NUREG/CR-6500, Rev. 1

ORNL/TM-13297/R1

-

\title{
Owners of Nuclear Power Plants
}

Prepared by

R. L. Reid, V. S. White

Oak Ridge National Laboratory

Prepared for

U.S. Nuclear Regulatory Commission

Washington, D.C. 20555 

NUREG/CR-6500, Rev. 1

ORNL/TM-13297/R1

\section{Owners of Nuclear Power Plants}

Manuscript Completed: January 2000

Date Published: February 2000

Prepared by

R. L. Reid, V. S. White

Oak Ridge National Laboratory

Managed by Lockheed Martin Energy Research Corporation

P.O. Box 2009

Oak Ridge, TN 37831-8065

R. Wood, NRC Technical Monitor

Prepared for

Office of Nuclear Reactor Regulation

U.S. Nuclear Regulatory Commission

Washington, D.C. 20555

NRC Job Code J2436 



\begin{abstract}
Commercial nuclear power plants in this country can be owned by a number of separate entities, each with varying ownership proportions. Each of these owners may, in turn, have a parent/subsidiary relationship to other companies. In addition, the operator of the plant may be a different entity as well. This report provides a compilation on the owners/operators for all commercial power reactors in the United States. While the utility industry is currently experiencing changes in organizational structure which may affect nuclear plant ownership, the data in this report is current as of November 1999. The report is divided into sections representing different aspects of nuclear plant ownership.
\end{abstract}





\section{CONTENTS}

Page

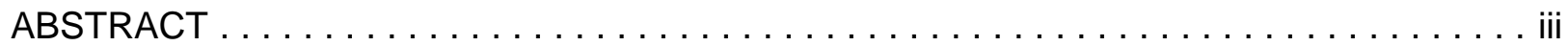

LIST OF TABLES $\ldots \ldots \ldots \ldots \ldots \ldots \ldots \ldots \ldots \ldots \ldots \ldots \ldots \ldots \ldots \ldots$ vii

ACKNOWLEDGMENTS $\ldots \ldots \ldots \ldots \ldots \ldots \ldots \ldots \ldots \ldots \ldots \ldots \ldots \ldots \ldots$

$1 \quad$ INTRODUCTION $\ldots \ldots \ldots \ldots \ldots \ldots \ldots \ldots \ldots \ldots \ldots \ldots \ldots \ldots \ldots$

2 NUCLEAR POWER PLANT PERCENTAGE OWNERSHIP ORDERED BY

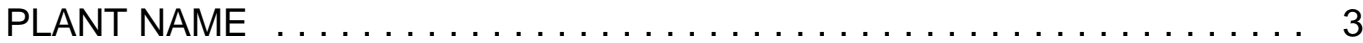

3 NUCLEAR POWER PLANT PERCENTAGE OWNERSHIP

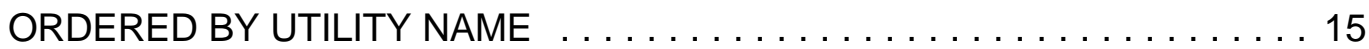

4 UTILITY/COMPANY RELATIONSHIPS ORDERED BY PARENT/HOLDING COMPANY ................. 29

5 UTILITY/COMPANY RELATIONSHIPS ORDERED BY SUBSIDIARY $\ldots \ldots \ldots . . .37$

$6 \quad$ NUCLEAR POWER PLANTS LISTED BY OPERATOR $\ldots \ldots \ldots \ldots \ldots \ldots$

$7 \quad$ NUCLEAR POWER PLANT OPERATORS LISTED BY PLANT NAME $\ldots \ldots . . .51$

$8 \quad$ NUCLEAR POWER PLANTS LISTED BY STATE $\ldots \ldots \ldots \ldots \ldots \ldots \ldots \ldots \ldots$

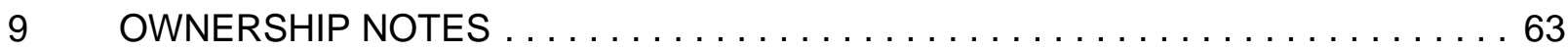

10 NUCLEAR POWER PLANT OPERATOR WEB SITES . . . . . . . . . . . 69

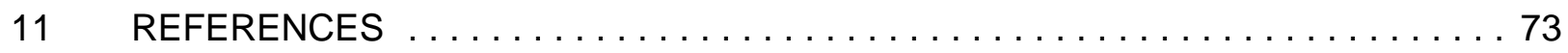





\section{LIST OF TABLES}

Table

$\underline{\text { Page }}$

1. Nuclear Power Plant Percentage Ownership by Plant $\ldots \ldots \ldots \ldots \ldots \ldots \ldots$

2. Nuclear Power Plant Percentage Ownership by Utility . . . . . . . . . . . 17

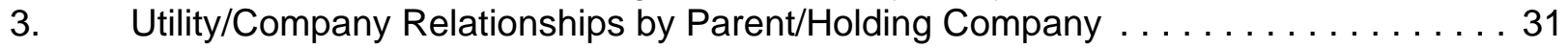

4. Utility/Company Relationships by Subsidiary $\ldots \ldots \ldots \ldots \ldots \ldots \ldots \ldots \ldots$

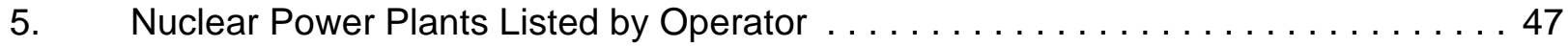

6. Nuclear Power Plant Operators Listed by Plant $\ldots \ldots \ldots \ldots \ldots \ldots \ldots \ldots \ldots$

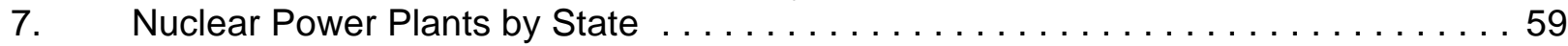

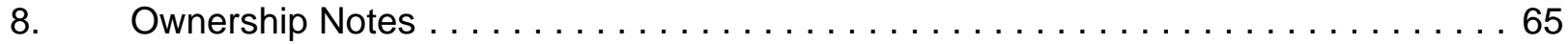

9. Nuclear Power Plant Operator Web Sites $\ldots \ldots \ldots \ldots \ldots \ldots \ldots \ldots \ldots \ldots$ 



\section{ACKNOWLEDGMENTS}

The authors wish to thank the utility company personnel who were contacted in order to assist in determining and/or confirming the ownership data contained in this report. The authors also wish to thank Amy Bush for her assistance in compiling the ownership data and preparing the report tables. In addition, the authors express their appreciation to Robert Wood, Technical Monitor, Division of Reactor Improvement Programs, Office of Nuclear Reactor Regulation, U.S. Nuclear Regulatory Commission, for his insight and guidance. 



\section{INTRODUCTION}

Commercial nuclear power plants in this country can be owned by a number of separate entities, each with varying ownership proportions. Each of these owners may, in turn, have a parent/subsidiary relationship to other companies. In addition, the operator of the plant may be a different entity as well. This report provides a compilation on the owners/operators for all commercial power reactors in the United States. While the utility industry is currently experiencing changes in organizational structure which may affect nuclear plant ownership, the data in this report is current as of November 1999. The report is divided into sections representing different aspects of nuclear plant ownership. 



\section{NUCLEAR POWER PLANT PERCENTAGE OWNERSHIP ORDERED BY PLANT NAME}



Table 1. Nuclear Power Plant Percentage Ownership by Plant

\begin{tabular}{|c|c|c|c|c|}
\hline Plant Name & Location & $\begin{array}{l}\text { Operating } \\
\text { Status }\end{array}$ & Ownership & $\begin{array}{l}\text { Ownership } \\
\text { Percentage }\end{array}$ \\
\hline Arkansas Nuclear One, Units $1 \& 2$ & Arkansas & FPL & Entergy Arkansas, Inc. & 100.0 \\
\hline Arnold (Duane) Energy Center & lowa & FPL & $\begin{array}{l}\text { IES Utilities, Inc. } \\
\text { Central lowa Power Cooperative } \\
\text { Corn Belt Power Cooperative }\end{array}$ & $\begin{array}{l}70.0 \\
20.0 \\
10.0\end{array}$ \\
\hline Beaver Valley Power Station, Unit 1 & Pennsylvania & $\mathrm{FPL}$ & $\begin{array}{l}\text { Pennsylvania Power Company } \\
\text { Ohio Edison Company }\end{array}$ & $\begin{array}{l}65.0 \\
35.0\end{array}$ \\
\hline Beaver Valley Power Station, Unit 2 & Pennsylvania & $\mathrm{FPL}$ & $\begin{array}{l}\text { Ohio Edison Company } \\
\text { Cleveland Electric Illuminating Company } \\
\text { Toledo Edison Company } \\
\text { Pennsylvania Power Company }\end{array}$ & $\begin{array}{l}41.88 \\
24.47 \\
19.91 \\
13.74\end{array}$ \\
\hline Bellefonte Nuclear Plant, Units $1 \& 2$ & Alabama & $\mathrm{CDH}$ & Tennessee Valley Authority & 100.0 \\
\hline Big Rock Point Nuclear Plant & Michigan & POL & Consumers Energy & 100.0 \\
\hline Braidwood Station, Units $1 \& 2$ & Illinois & FPL & Commonwealth Edison Company & 100.0 \\
\hline Browns Ferry Nuclear Power Station, Unit 1 & Alabama & SDUR & Tennessee Valley Authority & 100.0 \\
\hline Browns Ferry Nuclear Power Station, Units 2 \& 3 & Alabama & $\mathrm{FPL}$ & Tennessee Valley Authority & 100.0 \\
\hline Brunswick Steam Electric Plant, Units 1 \& 2 & North Carolina & $\mathrm{FPL}$ & $\begin{array}{l}\text { Carolina Power \& Light Company } \\
\text { North Carolina Eastern Municipal Power } \\
\quad \text { Agency }\end{array}$ & $\begin{array}{l}81.67 \\
18.33\end{array}$ \\
\hline Byron Station, Units $1 \& 2$ & Illinois & FPL & Commonwealth Edison Company & 100.0 \\
\hline Callaway Plant & Missouri & FPL & AmerenUE & 100.0 \\
\hline Calvert Cliffs Nuclear Power Plant, Units 1 \& 2 & Maryland & FPL & Baltimore Gas \& Electric Company & 100.0 \\
\hline
\end{tabular}


Table 1. Nuclear Power Plant Percentage Ownership by Plant

\begin{tabular}{|c|c|c|c|c|}
\hline Plant Name & Location & $\begin{array}{l}\text { Operating } \\
\text { Status }\end{array}$ & Ownership & $\begin{array}{l}\text { Ownership } \\
\text { Percentage }\end{array}$ \\
\hline Catawba Nuclear Station, Unit 1 & South Carolina & $\mathrm{FPL}$ & $\begin{array}{l}\text { North Carolina Electric Membership } \\
\quad \text { Corporation } \\
\text { Duke Energy Corporation } \\
\text { Saluda River Electric Cooperative }\end{array}$ & $\begin{array}{l}56.25 \\
25.00 \\
18.75\end{array}$ \\
\hline Catawba Nuclear Station, Unit 2 & South Carolina & FPL & $\begin{array}{l}\text { North Carolina Eastern Municipal Power } \\
\text { Agency } \\
\text { Piedmont Municipal Power Agency }\end{array}$ & $\begin{array}{l}75.0 \\
25.0\end{array}$ \\
\hline Clinton Power Station & Illinois & $\mathrm{FPL}$ & AmerGen Energy Company & 100.0 \\
\hline Comanche Peak Steam Electric Station, Units 1 \& 2 & Texas & $\mathrm{FPL}$ & TXU Electric and Gas & 100.0 \\
\hline Cook (Donald C.) Nuclear Power Plant, Units 1 \& 2 & Michigan & $\mathrm{FPL}$ & Indiana Michigan Power Company & 100.0 \\
\hline Cooper Nuclear Station & Nebraska & FPL & Nebraska Public Power District & 100.0 \\
\hline Crystal River Nuclear Plant, Unit 3 & Florida & FPL & $\begin{array}{l}\text { Florida Power Corporation } \\
\text { Seminole Electric Cooperative, Inc. } \\
\text { Orlando Utilities Commission } \\
\text { Gainesville (City of) Regional Utilities } \\
\text { Ocala (City of) Utilities Division } \\
\text { Leesburg (City of) Municipal Electric } \\
\text { Department } \\
\text { Kissimmee (City of) Utilities } \\
\text { New Smyrna Beach Utilities } \\
\text { Commission } \\
\text { Alachua (City of) Electric Department } \\
\text { Bushnell (City of) Utility Department }\end{array}$ & $\begin{array}{r}91.7806 \\
1.6994 \\
1.6015 \\
1.4079 \\
1.3333 \\
0.8244 \\
\\
0.6754 \\
0.5608 \\
\\
0.0779 \\
0.0388\end{array}$ \\
\hline Davis-Besse Nuclear Power Station & Ohio & FPL & $\begin{array}{l}\text { Cleveland Electric Illuminating Company } \\
\text { Toledo Edison Company }\end{array}$ & $\begin{array}{l}51.38 \\
48.62\end{array}$ \\
\hline Diablo Canyon Nuclear Power Plant, Units $1 \& 2$ & California & FPL & Pacific Gas \& Electric Company & 100.0 \\
\hline Dresden Nuclear Power Station, Unit 1 & Illinois & POL & Commonwealth Edison Company & 100.0 \\
\hline
\end{tabular}


Table 1. Nuclear Power Plant Percentage Ownership by Plant

\begin{tabular}{|c|c|c|c|c|}
\hline Plant Name & Location & $\begin{array}{l}\text { Operating } \\
\text { Status }\end{array}$ & Ownership & $\begin{array}{l}\text { Ownership } \\
\text { Percentage }\end{array}$ \\
\hline Dresden Nuclear Power Station, Units 2 \& 3 & Illinois & FPL & Commonwealth Edison Company & 100.0 \\
\hline Farley (Joseph M.) Nuclear Plant, Units $1 \& 2$ & Alabama & FPL & Alabama Power Company & 100.0 \\
\hline Fermi (Enrico) Atomic Power Plant, Unit 1 & Michigan & POL & Power Reactor Development Company & 100.0 \\
\hline Fermi (Enrico) Atomic Power Plant, Unit 2 & Michigan & $\mathrm{FPL}$ & Detroit Edison Company & 100.0 \\
\hline Fitzpatrick (James A.) Nuclear Power Plant & New York & FPL & New York Power Authority & 100.0 \\
\hline Fort Calhoun Station & Nebraska & FPL & Omaha Public Power District & 100.0 \\
\hline Fort St. Vrain Nuclear Generating Station & Colorado & LT & New Century Energies & 100.0 \\
\hline Ginna (Robert E.) Nuclear Power Plant & New York & $\mathrm{FPL}$ & Rochester Gas \& Electric Corporation & 100.0 \\
\hline Grand Gulf Nuclear Station & Mississippi & $\mathrm{FPL}$ & $\begin{array}{l}\text { System Energy Resources, Inc. } \\
\text { South Mississippi Electric Power } \\
\text { Association }\end{array}$ & $\begin{array}{l}90.0 \\
10.0\end{array}$ \\
\hline Haddam Neck (Connecticut Yankee) Plant & Connecticut & POL & $\begin{array}{l}\text { Connecticut Yankee Atomic Power } \\
\text { Company }\end{array}$ & 100.0 \\
\hline Harris (Shearon) Nuclear Power Plant & North Carolina & FPL & $\begin{array}{l}\text { Carolina Power \& Light Company } \\
\text { North Carolina Eastern Municipal Power } \\
\text { Agency }\end{array}$ & $\begin{array}{l}83.83 \\
16.17\end{array}$ \\
\hline Hatch (Edwin I.) Nuclear Plant, Units 1 \& 2 & Georgia & FPL & $\begin{array}{l}\text { Georgia Power Company } \\
\text { Oglethorpe Power Corporation } \\
\text { Municipal Electric Authority of Georgia } \\
\text { Dalton Water \& Light Sinking Fund } \\
\quad \text { Commission }\end{array}$ & $\begin{array}{r}50.1 \\
30.0 \\
17.7 \\
2.2\end{array}$ \\
\hline Hope Creek Nuclear Generating Station & New Jersey & FPL & $\begin{array}{l}\text { Public Service Electric \& Gas Company } \\
\text { Conectiv Inc. }\end{array}$ & $\begin{array}{r}95 \\
5.0\end{array}$ \\
\hline
\end{tabular}


Table 1. Nuclear Power Plant Percentage Ownership by Plant

\begin{tabular}{|c|c|c|c|c|}
\hline Plant Name & Location & $\begin{array}{l}\text { Operating } \\
\text { Status }\end{array}$ & Ownership & $\begin{array}{l}\text { Ownership } \\
\text { Percentage }\end{array}$ \\
\hline Humboldt Bay Power Plant, Unit 3 & California & POL & Pacific Gas \& Electric Company & 100.0 \\
\hline
\end{tabular}

Indian Poi92 481.441f 659.76 6.04 TD 5840144 Tk00.Øork Tw (California) Tj 90.72 0 TD -0.TD 0.0766 Tc (POL) Tj 623.04 TD039.0381 Consolidated Edison G 
Table 1. Nuclear Power Plant Percentage Ownership by Plant

\begin{tabular}{|c|c|c|c|c|}
\hline Plant Name & Location & $\begin{array}{l}\text { Operating } \\
\text { Status }\end{array}$ & Ownership & $\begin{array}{l}\text { Ownership } \\
\text { Percentage }\end{array}$ \\
\hline \multirow[t]{12}{*}{ Millstone Nuclear Power Station, Unit 3 (continued) } & & FPL & $\begin{array}{l}\text { Massachusetts Municipal Wholesale } \\
\text { Electric Company }\end{array}$ & 4.7990 \\
\hline & & & Montaup Electric Company & 4.0090 \\
\hline & & & United Illuminating Company & 3.6850 \\
\hline & & & $\begin{array}{l}\text { Public Service Company of New } \\
\text { Hampshire }\end{array}$ & 2.8475 \\
\hline & & & Central Maine Power Company & 2.5000 \\
\hline & & & $\begin{array}{l}\text { Central Vermont Public Service } \\
\text { Corporation }\end{array}$ & 1.7303 \\
\hline & & & Chicopee Electric Light Department & 1.3500 \\
\hline & & & $\begin{array}{l}\text { Connecticut Municipal Electric Energy } \\
\text { Cooperative. Inc. }\end{array}$ & 1.0870 \\
\hline & & & Vermont Electric Generation \& & 0.3500 \\
\hline & & & Transmission Cooperative, Inc. & 2170 \\
\hline & & & Fitchburg Gas \& Electric Company & $\begin{array}{l}0.2170 \\
0.0487\end{array}$ \\
\hline & & & Department & \\
\hline Monticello Nuclear Generating Plant & Minnesota & FPL & Northern States Power Company & 100.0 \\
\hline Nine Mile Point Nuclear Station, Unit 1 & New York & FPL & Niagara Mohawk Power Corporation & 100.0 \\
\hline \multirow[t]{5}{*}{ Nine Mile Point Nuclear Station, Unit 2} & New York & FPL & Niagara Mohawk Power Corporation & 41.0 \\
\hline & & & Long Island Power Authority & 18.0 \\
\hline & & & $\begin{array}{l}\text { New York State Electric \& Gas } \\
\text { Corporation }\end{array}$ & 18.0 \\
\hline & & & Rochester Gas \& Electric Corporation & 14.0 \\
\hline & & & $\begin{array}{l}\text { Central Hudson Gas \& Electric } \\
\text { Corporation }\end{array}$ & 9.0 \\
\hline \multirow[t]{2}{*}{ North Anna Power Station, Units $1 \& 2$} & Virginia & FPL & Virginia Electric and Power Company & 88.4 \\
\hline & & & Old Dominion Electric Cooperative & 11.6 \\
\hline Oconee Nuclear Station, Units $1-3$ & South Carolina & FPL & Duke Energy Corporation & 100.0 \\
\hline Oyster Creek Nuclear Power Plant & New Jersey & FPL & Jersey Central Power \& Light Company & 100.0 \\
\hline
\end{tabular}


Table 1. Nuclear Power Plant Percentage Ownership by Plant

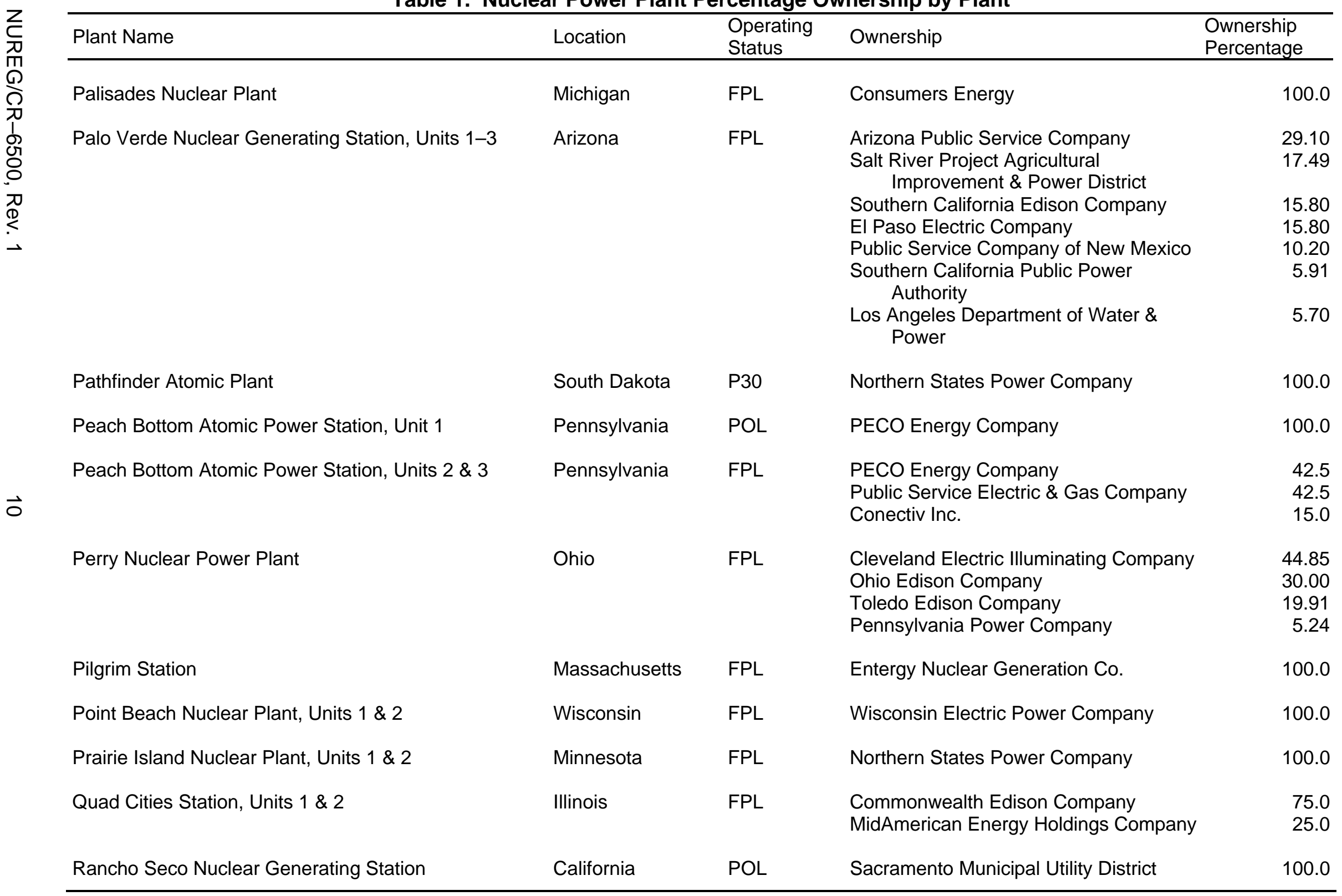


Table 1. Nuclear Power Plant Percentage Ownership by Plant

\begin{tabular}{|c|c|c|c|c|}
\hline Plant Name & Location & $\begin{array}{l}\text { Operating } \\
\text { Status }\end{array}$ & Ownership & $\begin{array}{l}\text { Ownership } \\
\text { Percentage }\end{array}$ \\
\hline River Bend Station & Louisiana & FPL & Entergy Gulf States, Inc. & 100.0 \\
\hline Robinson (H. B.) Plant, Unit 2 & South Carolina & FPL & Carolina Power \& Light Company & 100.0 \\
\hline Salem Nuclear Generating Station, Units 1 \& 2 & New Jersey & FPL & $\begin{array}{l}\text { Public Service Electric \& Gas Company } \\
\text { PECO Energy Company } \\
\text { Conectiv Inc. }\end{array}$ & $\begin{array}{l}42.59 \\
42.59 \\
14.82\end{array}$ \\
\hline San Onofre Nuclear Generating Station, Unit 1 & California & POL & $\begin{array}{l}\text { Southern California Edison Company } \\
\text { San Diego Gas \& Electric Company }\end{array}$ & $\begin{array}{l}80.0 \\
20.0\end{array}$ \\
\hline San Onofre Nuclear Generating Station, Units 2 \& 3 & California & FPL & $\begin{array}{l}\text { Southern California Edison Company } \\
\text { San Diego Gas \& Electric Company } \\
\text { Anaheim Public Utilities Department } \\
\text { Riverside Utilities Department }\end{array}$ & $\begin{array}{r}75.05 \\
20.0 \\
3.16 \\
1.79\end{array}$ \\
\hline Saxton Nuclear Experimental Reactor Project & Pennsylvania & POL & GPU, Inc. & 100.0 \\
\hline Seabrook Nuclear Power Station & New Hampshire & FPL & $\begin{array}{l}\text { North Atlantic Energy Corporation } \\
\text { United Illuminating Company } \\
\text { Great Bay Power Corporation } \\
\text { Massachusetts Municipal Wholesale } \\
\text { Electric Company } \\
\text { New England Power Company } \\
\text { Connecticut Light \& Power Company } \\
\text { Canal Electric Company } \\
\text { Little Bay Power Corporation } \\
\text { New Hampshire Electric Cooperative, } \\
\text { Inc. } \\
\text { Taunton Municipal Light Plant } \\
\text { Hudson Light \& Power Department }\end{array}$ & $\begin{array}{r}35.98201 \\
17.50000 \\
12.13240 \\
11.59340 \\
\\
9.95766 \\
4.05985 \\
3.52317 \\
2.89989 \\
2.17391 \\
\\
0.10034 \\
0.07737\end{array}$ \\
\hline Sequoyah Nuclear Plant, Units $1 \& 2$ & Tennessee & FPL & Tennessee Valley Authority & 100.0 \\
\hline Shoreham Nuclear Power Station & New York & LT & Long Island Power Authority & 100.0 \\
\hline
\end{tabular}


Table 1. Nuclear Power Plant Percentage Ownership by Plant

\begin{tabular}{|c|c|c|c|c|}
\hline Plant Name & Location & $\begin{array}{l}\text { Operating } \\
\text { Status }\end{array}$ & Ownership & $\begin{array}{l}\text { Ownership } \\
\text { Percentage }\end{array}$ \\
\hline South Texas Project, Units $1 \& 2$ & Texas & FPL & $\begin{array}{l}\text { Reliant Energy HL\&P } \\
\text { San Antonio City Public Service Board } \\
\text { Central Power \& Light } \\
\text { Austin Electric Department }\end{array}$ & $\begin{array}{l}30.8 \\
28.0 \\
25.2 \\
16.0\end{array}$ \\
\hline St. Lucie Plant, Unit 1 & Florida & $\mathrm{FPL}$ & Florida Power \& Light Company & 100.0 \\
\hline St. Lucie Plant, Unit 2 & Florida & $\mathrm{FPL}$ & $\begin{array}{l}\text { Florida Power \& Light Company } \\
\text { Florida Municipal Power Agency } \\
\text { Orlando Utilities Commission }\end{array}$ & $\begin{array}{r}85.10449 \\
8.80600 \\
6.08951\end{array}$ \\
\hline Summer (Virgil C.) Nuclear Station & South Carolina & $\mathrm{FPL}$ & $\begin{array}{l}\text { South Carolina Electric \& Gas Company } \\
\text { South Carolina Public Service Authority }\end{array}$ & $\begin{array}{l}66.667 \\
33.333\end{array}$ \\
\hline Surry Power Station, Units $1 \& 2$ & Virginia & FPL & Virginia Electric and Power Company & 100.0 \\
\hline Susquehanna Steam Electric Station, Units 1 \& 2 & Pennsylvania & $\mathrm{FPL}$ & $\begin{array}{l}\text { PP\&L, Inc. } \\
\text { Allegheny Electric Cooperative, Inc. }\end{array}$ & $\begin{array}{l}90.0 \\
10.0\end{array}$ \\
\hline Three Mile Island Nuclear Station, Unit 1 & Pennsylvania & FPL & AmerGen Energy Company & 100.0 \\
\hline Three Mile Island Nuclear Station, Unit 2 & Pennsylvania & POL & $\begin{array}{l}\text { Metropolitan Edison Company } \\
\text { Pennsylvania Electric Company } \\
\text { Jersey Central Power \& Light Company }\end{array}$ & $\begin{array}{l}50.0 \\
25.0 \\
25.0\end{array}$ \\
\hline Trojan Nuclear Plant & Oregon & POL & $\begin{array}{l}\text { Portland General Electric Company } \\
\text { Eugene Water \& Electric Board } \\
\text { Pacific Power \& Light Company }\end{array}$ & $\begin{array}{r}67.5 \\
30.0 \\
2.5\end{array}$ \\
\hline Turkey Point Station, Unit 3 \& 4 & Florida & FPL & Florida Power \& Light Company & 100.0 \\
\hline Vallecitos Boiling Water Reactor & California & POL & General Electric Company & 100.0 \\
\hline Vermont Yankee Nuclear Power Station & Vermont & $\mathrm{FPL}$ & $\begin{array}{l}\text { Vermont Yankee Nuclear Power } \\
\text { Corporation }\end{array}$ & 100.0 \\
\hline
\end{tabular}


Table 1. Nuclear Power Plant Percentage Ownership by Plant

\begin{tabular}{|c|c|c|c|c|}
\hline Plant Name & Location & $\begin{array}{l}\text { Operating } \\
\text { Status }\end{array}$ & Ownership & $\begin{array}{l}\text { Ownership } \\
\text { Percentage }\end{array}$ \\
\hline Vogtle (Alvin W.) Nuclear Plant, Units $1 \& 2$ & Georgia & FPL & $\begin{array}{l}\text { Georgia Power Company } \\
\text { Oglethorpe Power Corporation } \\
\text { Municipal Electric Authority of Georgia } \\
\text { Dalton Water \& Light Sinking Fund } \\
\quad \text { Commission }\end{array}$ & $\begin{array}{r}45.7 \\
30.0 \\
22.7 \\
1.6\end{array}$ \\
\hline Waterford Generating Station, Unit 3 & Louisiana & FPL & Entergy Louisiana, Inc. & 100.0 \\
\hline Watts Bar Nuclear Plant, Unit 1 & Tennessee & FPL & Tennessee Valley Authority & 100.0 \\
\hline Watts Bar Nuclear Plant, Unit 2 & Tennessee & $\mathrm{CDH}$ & Tennessee Valley Authority & 100.0 \\
\hline WNP-2 & Washington & FPL & Energy Northwest & 100.0 \\
\hline Wolf Creek Generating Station & Kansas & FPL & $\begin{array}{l}\text { Kansas Gas \& Electric Company } \\
\text { Kansas City Power \& Light Company } \\
\text { Kansas Electric Power Cooperative, Inc. }\end{array}$ & $\begin{array}{r}47.0 \\
47.0 \\
6.0\end{array}$ \\
\hline WPPSS Nuclear Project, Unit 1 & Washington & $\mathrm{CDH}$ & Energy Northwest & 100.0 \\
\hline WPPSS Nuclear Project, Unit 3 & Washington & $\mathrm{CDH}$ & $\begin{array}{l}\text { Energy Northwest } \\
\text { Portland General Electric Company } \\
\text { Pacific Power \& Light Company } \\
\text { Avista Utilities } \\
\text { Puget Sound Energy }\end{array}$ & $\begin{array}{r}70.0 \\
10.0 \\
10.0 \\
5.0 \\
5.0\end{array}$ \\
\hline Yankee Nuclear Power Station & Massachusetts & POL & Yankee Atomic Electric Company & 100.0 \\
\hline Zion Nuclear Plant, Units 1 \& 2 & Illinois & POL & Commonwealth Edison Company & 100.0 \\
\hline $\begin{array}{l}\text { Operating Status Codes: } \\
C D H \text { - construction deferred or halted } \\
F P L-\text { full power license } \\
L T \text { - license terminated }\end{array}$ & \multicolumn{4}{|c|}{$\begin{array}{l}P O L-\text { possession only license } \\
\text { P30 - Part } 30, \text { materials possession only license } \\
\text { SDUR - shut down; under review }\end{array}$} \\
\hline
\end{tabular}





\section{NUCLEAR POWER PLANT PERCENTAGE OWNERSHIP ORDERED BY UTILITY NAME}



Table 2. Nuclear Power Plant Percentage Ownership by Utility

\begin{tabular}{|c|c|c|}
\hline Utility & Plant Name & $\begin{array}{l}\text { Ownership } \\
\text { Percentage }\end{array}$ \\
\hline Alabama Power Company & Farley (Joseph M.) Nuclear Plant, Units $1 \& 2$ & 100.0 \\
\hline Alachua (City of) Electric Department & Crystal River Nuclear Plant, Unit 3 & 0.0779 \\
\hline Allegheny Electric Cooperative, Inc. & Susquehanna Steam Electric Station, Units 1 \& 2 & 10.0 \\
\hline AmerenUE & Callaway Plant & 100.0 \\
\hline \multirow[t]{2}{*}{ AmerGen Energy Company } & Clinton Power Station & 100.0 \\
\hline & Three Mile Island Nuclear Station, Unit 1 & 100.0 \\
\hline Anaheim Public Utilities Department & San Onofre Nuclear Generating Station, Units 2 \& 3 & 3.16 \\
\hline Arizona Public Service Company & Palo Verde Nuclear Generating Station, Units 1-3 & 29.1 \\
\hline Austin Electric Department & South Texas Project, Units $1 \& 2$ & 16.0 \\
\hline Avista Utilities & WPPSS Nuclear Project, Unit 3 & 5.0 \\
\hline Baltimore Gas \& Electric Company & Calvert Cliffs Nuclear Power Plant, Units 1 \& 2 & 100.0 \\
\hline Bushnell (City of) Utility Department & Crystal River Nuclear Plant, Unit 3 & 0.0388 \\
\hline Canal Electric Company & Seabrook Nuclear Power Station & 3.52317 \\
\hline \multirow[t]{3}{*}{ Carolina Power \& Light Company } & Brunswick Steam Electric Plant, Units $1 \& 2$ & 81.67 \\
\hline & Harris (Shearon) Nuclear Power Plant & 83.83 \\
\hline & Robinson (H. B.) Plant, Unit 2 & 100.0 \\
\hline Central Hudson Gas \& Electric Corporation & Nine Mile Point Nuclear Station, Unit 2 & 9.0 \\
\hline
\end{tabular}


Table 2. Nuclear Power Plant Percentage Ownership by Utility

\begin{tabular}{|c|c|c|}
\hline Utility & Plant Name & $\begin{array}{l}\text { Ownership } \\
\text { Percentage }\end{array}$ \\
\hline Central lowa Power Cooperative & Arnold (Duane) Energy Center & 20.0 \\
\hline Central Maine Power Company & Millstone Nuclear Power Station, Unit 3 & 2.5 \\
\hline Central Power \& Light & South Texas Project, Units $1 \& 2$ & 25.2 \\
\hline Central Vermont Public Service Corporation & Millstone Nuclear Power Station, Unit 3 & 1.7303 \\
\hline Chicopee Electric Light Department & Millstone Nuclear Power Station, Unit 3 & 1.35 \\
\hline \multirow[t]{3}{*}{ Cleveland Electric Illuminating Company } & Beaver Valley Power Station, Unit 2 & 24.47 \\
\hline & Davis-Besse Nuclear Power Station & 51.38 \\
\hline & Perry Nuclear Power Plant & 44.85 \\
\hline \multirow[t]{7}{*}{ Commonwealth Edison Company } & Braidwood Station, Units $1 \& 2$ & 100.0 \\
\hline & Byron Station, Units $1 \& 2$ & 100.0 \\
\hline & Dresden Nuclear Power Station, Unit 1 & 100.0 \\
\hline & Dresden Nuclear Power Station, Units 2 \& 3 & 100.0 \\
\hline & LaSalle County Station, Units $1 \& 2$ & 100.0 \\
\hline & Quad Cities Station, Units 1 \& 2 & 75.0 \\
\hline & Zion Nuclear Plant, Units 1 \& 2 & 100.0 \\
\hline \multirow[t]{3}{*}{ Conectiv Inc. } & Hope Creek Nuclear Generating Station & 5.00 \\
\hline & Peach Bottom Atomic Power Station, Units 2 \& 3 & 15.00 \\
\hline & Salem Nuclear Generating Station, Units 1 \& 2 & 14.82 \\
\hline \multirow[t]{4}{*}{ Connecticut Light \& Power Company } & Millstone Nuclear Power Station, Unit 1 & 80.00000 \\
\hline & Millstone Nuclear Power Station, Unit 2 & 80.00000 \\
\hline & Millstone Nuclear Power Station, Unit 3 & 52.93300 \\
\hline & Seabrook Nuclear Power Station & 4.05985 \\
\hline
\end{tabular}


Table 2. Nuclear Power Plant Percentage Ownership by Utility

\begin{tabular}{|c|c|c|}
\hline Utility & Plant Name & $\begin{array}{l}\text { Ownership } \\
\text { Percentage }\end{array}$ \\
\hline Connecticut Municipal Electric Energy Cooperative, Inc. & Millstone Nuclear Power Station, Unit 3 & 1.087 \\
\hline Connecticut Yankee Atomic Power Company & Haddam Neck (Connecticut Yankee) Plant & 100.0 \\
\hline \multirow[t]{2}{*}{ Consolidated Edison Company of New York } & Indian Point, Unit 1 & 100.0 \\
\hline & Indian Point, Unit 2 & 100.0 \\
\hline \multirow[t]{2}{*}{ Consumers Energy } & Big Rock Point Nuclear Plant & 100.0 \\
\hline & Palisades Nuclear Plant & 100.0 \\
\hline Corn Belt Power Cooperative & Arnold (Duane) Energy Center & 10.0 \\
\hline Dairyland Power Cooperative & LaCrosse (Genoa) Nuclear Generating Station & 100.0 \\
\hline \multirow[t]{2}{*}{ Dalton Water \& Light Sinking Fund Commission } & Hatch (Edwin I.) Nuclear Plant, Units 1 \& 2 & 2.2 \\
\hline & Vogtle (Alvin W.) Nuclear Plant, Units $1 \& 2$ & 1.6 \\
\hline Detroit Edison Company & Fermi (Enrico) Atomic Power Plant, Unit 2 & 100.0 \\
\hline \multirow[t]{3}{*}{ Duke Energy Corporation } & Catawba Nuclear Station, Unit 1 & 25.0 \\
\hline & McGuire (William B.) Nuclear Station, Units 1 \& 2 & 100.0 \\
\hline & Oconee Nuclear Station, Units $1-3$ & 100.0 \\
\hline El Paso Electric Company & Palo Verde Nuclear Generating Station, Units 1-3 & 15.8 \\
\hline \multirow[t]{3}{*}{ Energy Northwest } & WNP-2 & 100.0 \\
\hline & WPPSS Nuclear Project, Unit 1 & 100.0 \\
\hline & WPPSS Nuclear Project, Unit 3 & 70.0 \\
\hline Entergy Arkansas, Inc. & Arkansas Nuclear One, Units $1 \& 2$ & 100.0 \\
\hline
\end{tabular}


Table 2. Nuclear Power Plant Percentage Ownership by Utility

\begin{tabular}{|c|c|c|}
\hline Utility & Plant Name & $\begin{array}{l}\text { Ownership } \\
\text { Percentage }\end{array}$ \\
\hline Entergy Gulf States, Inc. & River Bend Station & 100.0 \\
\hline Entergy Louisiana, Inc. & Waterford Generating Station, Unit 3 & 100.0 \\
\hline Entergy Nuclear Generation Co. & Pilgrim Station & 100.0 \\
\hline Eugene Water \& Electric Board & Trojan Nuclear Plant & 30.0 \\
\hline Fitchburg Gas \& Electric Company & Millstone Nuclear Power Station, Unit 3 & 0.217 \\
\hline Florida Municipal Power Agency & St. Lucie Plant, Unit 2 & 8.806 \\
\hline \multirow[t]{3}{*}{ Florida Power \& Light Company } & St. Lucie Plant, Unit 1 & 100.00000 \\
\hline & St. Lucie Plant, Unit 2 & 85.10449 \\
\hline & Turkey Point Station, Unit 3 \& 4 & 100.00000 \\
\hline Florida Power Corporation & Crystal River Nuclear Plant, Unit 3 & 91.7806 \\
\hline Gainesville (City of) Regional Utilities & Crystal River Nuclear Plant, Unit 3 & 1.4079 \\
\hline General Electric Company & Vallecitos Boiling Water Reactor & 100.0 \\
\hline \multirow[t]{2}{*}{ Georgia Power Company } & Hatch (Edwin I.) Nuclear Plant, Units 1 \& 2 & 50.1 \\
\hline & Vogtle (Alvin W.) Nuclear Plant, Units 1 \& 2 & 45.7 \\
\hline GPU, Inc. & Saxton Nuclear Experimental Reactor Project & 100.0 \\
\hline Great Bay Power Corporation & Seabrook Nuclear Power Station & 12.1324 \\
\hline Hudson Light \& Power Department & Seabrook Nuclear Power Station & 0.07737 \\
\hline
\end{tabular}


Table 2. Nuclear Power Plant Percentage Ownership by Utility

\begin{tabular}{|c|c|c|}
\hline Utility & Plant Name & $\begin{array}{l}\text { Ownership } \\
\text { Percentage }\end{array}$ \\
\hline IES Utilities, Inc. & Arnold (Duane) Energy Center & 70.0 \\
\hline Indiana Michigan Power Company & Cook (Donald C.) Nuclear Power Plant, Units 1 \& 2 & 100.0 \\
\hline \multirow[t]{2}{*}{ Jersey Central Power \& Light Company } & Oyster Creek Nuclear Power Plant & 100.0 \\
\hline & Three Mile Island Nuclear Station, Unit 2 & 25.0 \\
\hline Kansas City Power \& Light Company & Wolf Creek Generating Station & 47.0 \\
\hline Kansas Electric Power Cooperative, Inc. & Wolf Creek Generating Station & 6.0 \\
\hline Kansas Gas \& Electric Company & Wolf Creek Generating Station & 47.0 \\
\hline Kissimmee (City of) Utilities & Crystal River Nuclear Plant, Unit 3 & 0.6754 \\
\hline Leesburg (City of) Municipal Electric Department & Crystal River Nuclear Plant, Unit 3 & 0.8244 \\
\hline Little Bay Power Corporation & Seabrook Nuclear Power Station & 2.89989 \\
\hline \multirow[t]{2}{*}{ Long Island Power Authority } & Nine Mile Point Nuclear Station, Unit 2 & 18.0 \\
\hline & Shoreham Nuclear Power Station & 100.0 \\
\hline Los Angeles Department of Water \& Power & Palo Verde Nuclear Generating Station, Units 1-3 & 5.7 \\
\hline Lyndonville (Village of) Electric Department & Millstone Nuclear Power Station, Unit 3 & 0.0487 \\
\hline Madison Gas \& Electric Company & Kewaunee Nuclear Power Plant & 17.8 \\
\hline Maine Yankee Atomic Power Company & Maine Yankee Atomic Power Station & 100.0 \\
\hline
\end{tabular}


Table 2. Nuclear Power Plant Percentage Ownership by Utility

\begin{tabular}{|c|c|c|}
\hline Utility & Plant Name & $\begin{array}{l}\text { Ownership } \\
\text { Percentage }\end{array}$ \\
\hline \multirow[t]{2}{*}{ Massachusetts Municipal Wholesale Electric Company } & Millstone Nuclear Power Station, Unit 3 & 4.7990 \\
\hline & Seabrook Nuclear Power Station & 11.5934 \\
\hline Metropolitan Edison Company & Three Mile Island Nuclear Station, Unit 2 & 50.0 \\
\hline MidAmerican Energy Holdings Company & Quad Cities Station, Units $1 \& 2$ & 25.0 \\
\hline Montaup Electric Company & Millstone Nuclear Power Station, Unit 3 & 4.009 \\
\hline \multirow[t]{2}{*}{ Municipal Electric Authority of Georgia } & Hatch (Edwin I.) Nuclear Plant, Units 1 \& 2 & 17.7 \\
\hline & Vogtle (Alvin W.) Nuclear Plant, Units $1 \& 2$ & 22.7 \\
\hline Nebraska Public Power District & Cooper Nuclear Station & 100.0 \\
\hline New Century Energies & Fort St. Vrain Nuclear Generating Station & 100.0 \\
\hline \multirow[t]{2}{*}{ New England Power Company } & Millstone Nuclear Power Station, Unit 3 & 12.20500 \\
\hline & Seabrook Nuclear Power Station & 9.95766 \\
\hline New Hampshire Electric Cooperative, Inc. & Seabrook Nuclear Power Station & 2.17391 \\
\hline New Smyrna Beach Utilities Commission & Crystal River Nuclear Plant, Unit 3 & 0.5608 \\
\hline \multirow[t]{2}{*}{ New York Power Authority } & Fitzpatrick (James A.) Nuclear Power Plant & 100.0 \\
\hline & Indian Point, Unit 3 & 100.0 \\
\hline New York State Electric \& Gas Corporation & Nine Mile Point Nuclear Station, Unit 2 & 18.0 \\
\hline \multirow[t]{2}{*}{ Niagara Mohawk Power Corporation } & Nine Mile Point Nuclear Station, Unit 1 & 100.0 \\
\hline & Nine Mile Point Nuclear Station, Unit 2 & 41.0 \\
\hline
\end{tabular}


Table 2. Nuclear Power Plant Percentage Ownership by Utility

\begin{tabular}{|c|c|c|}
\hline Utility & Plant Name & $\begin{array}{l}\text { Ownership } \\
\text { Percentage }\end{array}$ \\
\hline North Atlantic Energy Corporation & Seabrook Nuclear Power Station & 35.98201 \\
\hline \multirow[t]{3}{*}{ North Carolina Eastern Municipal Power Agency } & Brunswick Steam Electric Plant, Units $1 \& 2$ & 18.33 \\
\hline & Catawba Nuclear Station, Unit 2 & 75.00 \\
\hline & Harris (Shearon) Nuclear Power Plant & 16.17 \\
\hline North Carolina Electric Membership Corporation & Catawba Nuclear Station, Unit 1 & 56.25 \\
\hline \multirow[t]{3}{*}{ Northern States Power Company } & Monticello Nuclear Generating Plant & 100.0 \\
\hline & Pathfinder Atomic Plant & 100.0 \\
\hline & Prairie Island Nuclear Plant, Units $1 \& 2$ & 100.0 \\
\hline Ocala (City of) Utilities Division & Crystal River Nuclear Plant, Unit 3 & 1.3333 \\
\hline \multirow[t]{2}{*}{ Oglethorpe Power Corporation } & Hatch (Edwin I.) Nuclear Plant, Units 1 \& 2 & 30.0 \\
\hline & Vogtle (Alvin W.) Nuclear Plant, Units 1 \& 2 & 30.0 \\
\hline \multirow[t]{3}{*}{ Ohio Edison Company } & Beaver Valley Power Station, Unit 1 & 35.00 \\
\hline & Beaver Valley Power Station, Unit 2 & 41.88 \\
\hline & Perry Nuclear Power Plant & 30.00 \\
\hline Old Dominion Electric Cooperative & North Anna Power Station, Units $1 \& 2$ & 11.6 \\
\hline Omaha Public Power District & Fort Calhoun Station & 100.0 \\
\hline \multirow[t]{2}{*}{ Orlando Utilities Commission } & Crystal River Nuclear Plant, Unit 3 & 1.60150 \\
\hline & St. Lucie Plant, Unit 2 & 6.08951 \\
\hline \multirow[t]{2}{*}{ Pacific Gas \& Electric Company } & Diablo Canyon Nuclear Power Plant, Units 1 \& 2 & 100.0 \\
\hline & Humboldt Bay Power Plant, Unit 3 & 100.0 \\
\hline
\end{tabular}


Table 2. Nuclear Power Plant Percentage Ownership by Utility

\begin{tabular}{|c|c|c|}
\hline Utility & Plant Name & $\begin{array}{l}\text { Ownership } \\
\text { Percentage }\end{array}$ \\
\hline \multirow[t]{2}{*}{ Pacific Power \& Light Company } & Trojan Nuclear Plant & 2.5 \\
\hline & WPPSS Nuclear Project, Unit 3 & 10.0 \\
\hline \multirow[t]{4}{*}{ PECO Energy Company } & Limerick Generating Station, Units 1 \& 2 & 100.00 \\
\hline & Peach Bottom Atomic Power Station, Unit 1 & 100.00 \\
\hline & Peach Bottom Atomic Power Station, Units 2 \& 3 & 42.50 \\
\hline & Salem Nuclear Generating Station, Units 1 \& 2 & 42.59 \\
\hline Pennsylvania Electric Company & Three Mile Island Nuclear Station, Unit 2 & 25.0 \\
\hline \multirow[t]{3}{*}{ Pennsylvania Power Company } & Beaver Valley Power Station, Unit 1 & 65.00 \\
\hline & Beaver Valley Power Station, Unit 2 & 13.74 \\
\hline & Perry Nuclear Power Plant & 5.24 \\
\hline Piedmont Municipal Power Agency & Catawba Nuclear Station, Unit 2 & 25.0 \\
\hline \multirow[t]{2}{*}{ Portland General Electric Company } & Trojan Nuclear Plant & 67.5 \\
\hline & WPPSS Nuclear Project, Unit 3 & 10.0 \\
\hline Power Reactor Development Company & Fermi (Enrico) Atomic Power Plant, Unit 1 & 100.0 \\
\hline PP\&L, Inc. & Susquehanna Steam Electric Station, Units 1 \& 2 & 90.0 \\
\hline Public Service Company of New Hampshire & Millstone Nuclear Power Station, Unit 3 & 2.8475 \\
\hline Public Service Company of New Mexico & Palo Verde Nuclear Generating Station, Units 1-3 & 10.2 \\
\hline \multirow[t]{3}{*}{ Public Service Electric \& Gas Company } & Hope Creek Nuclear Generating Station & 95.00 \\
\hline & Peach Bottom Atomic Power Station, Units 2 \& 3 & 42.50 \\
\hline & Salem Nuclear Generating Station, Units 1 \& 2 & 42.59 \\
\hline
\end{tabular}


Table 2. Nuclear Power Plant Percentage Ownership by Utility

\begin{tabular}{|c|c|c|}
\hline Utility & Plant Name & $\begin{array}{l}\text { Ownership } \\
\text { Percentage }\end{array}$ \\
\hline Puget Sound Energy & WPPSS Nuclear Project, Unit 3 & 5.0 \\
\hline Reliant Energy HL\&P & South Texas Project, Units $1 \& 2$ & 30.8 \\
\hline Riverside Utilities Department & San Onofre Nuclear Generating Station, Units 2 \& 3 & 1.79 \\
\hline \multirow[t]{2}{*}{ Rochester Gas \& Electric Corporation } & Ginna (Robert E.) Nuclear Power Plant & 100.0 \\
\hline & Nine Mile Point Nuclear Station, Unit 2 & 14.0 \\
\hline Sacramento Municipal Utility District & Rancho Seco Nuclear Generating Station & 100.0 \\
\hline Salt River Project Agricultural Improvement \& Power District & Palo Verde Nuclear Generating Station, Units 1-3 & 17.49 \\
\hline Saluda River Electric Cooperative & Catawba Nuclear Station, Unit 1 & 18.75 \\
\hline San Antonio City Public Service Board & South Texas Project, Units $1 \& 2$ & 28.0 \\
\hline \multirow[t]{2}{*}{ San Diego Gas \& Electric Company } & San Onofre Nuclear Generating Station, Unit 1 & 20.0 \\
\hline & San Onofre Nuclear Generating Station, Units 2 \& 3 & 20.0 \\
\hline Seminole Electric Cooperative, Inc. & Crystal River Nuclear Plant, Unit 3 & 1.6994 \\
\hline South Carolina Electric \& Gas Company & Summer (Virgil C.) Nuclear Station & 66.667 \\
\hline South Carolina Public Service Authority & Summer (Virgil C.) Nuclear Station & 33.333 \\
\hline South Mississippi Electric Power Association & Grand Gulf Nuclear Station & 10.0 \\
\hline \multirow[t]{2}{*}{ Southern California Edison Company } & Palo Verde Nuclear Generating Station, Units 1-3 & 15.80 \\
\hline & San Onofre Nuclear Generating Station, Unit 1 & 80.00 \\
\hline
\end{tabular}


Table 2. Nuclear Power Plant Percentage Ownership by Utility

\begin{tabular}{|c|c|c|}
\hline Utility & Plant Name & $\begin{array}{l}\text { Ownership } \\
\text { Percentage }\end{array}$ \\
\hline Southern California Edison Company (continued) & San Onofre Nuclear Generating Station, Units 2 \& 3 & 75.05 \\
\hline Southern California Public Power Authority & Palo Verde Nuclear Generating Station, Units 1-3 & 5.91 \\
\hline System Energy Resources, Inc. & Grand Gulf Nuclear Station & 90.0 \\
\hline Taunton Municipal Light Plant & Seabrook Nuclear Power Station & 0.10034 \\
\hline \multirow[t]{6}{*}{ Tennessee Valley Authority } & Bellefonte Nuclear Plant, Units $1 \& 2$ & 100.0 \\
\hline & Browns Ferry Nuclear Power Station, Unit 1 & 100.0 \\
\hline & Browns Ferry Nuclear Power Station, Units 2 \& 3 & 100.0 \\
\hline & Sequoyah Nuclear Plant, Units $1 \& 2$ & 100.0 \\
\hline & Watts Bar Nuclear Plant, Unit 1 & 100.0 \\
\hline & Watts Bar Nuclear Plant, Unit 2 & 100.0 \\
\hline \multirow[t]{3}{*}{ Toledo Edison Company } & Beaver Valley Power Station, Unit 2 & 19.91 \\
\hline & Davis-Besse Nuclear Power Station & 48.62 \\
\hline & Perry Nuclear Power Plant & 19.91 \\
\hline TXU Electric and Gas & Comanche Peak Steam Electric Station, Units 1 \& 2 & 100.0 \\
\hline \multirow[t]{2}{*}{ United Illuminating Company } & Millstone Nuclear Power Station, Unit 3 & 3.685 \\
\hline & Seabrook Nuclear Power Station & 17.500 \\
\hline Vermont Electric Generation \& Transmission Cooperative, Inc. & Millstone Nuclear Power Station, Unit 3 & 0.35 \\
\hline Vermont Yankee Nuclear Power Corporation & Vermont Yankee Nuclear Power Station & 100.0 \\
\hline \multirow[t]{2}{*}{ Virginia Electric and Power Company } & North Anna Power Station, Units $1 \& 2$ & 88.4 \\
\hline & Surry Power Station, Units $1 \& 2$ & 100.0 \\
\hline
\end{tabular}


Table 2. Nuclear Power Plant Percentage Ownership by Utility

\begin{tabular}{llr}
\hline Utility & Plant Name & $\begin{array}{c}\text { Ownership } \\
\text { Percentage }\end{array}$ \\
\hline Western Massachusetts Electric Company & Millstone Nuclear Power Station, Unit 1 & $\begin{array}{r}20.0000 \\
20.0000 \\
12.2385\end{array}$ \\
Willstone Nuclear Power Station, Unit 2 & Millstone Nuclear Power Station, Unit 3 \\
Wisconsin Electric Power Company & Point Beach Nuclear Plant, Units 1\&2 \\
Wisconsin Public Service Corporation & Kewaunee Nuclear Power Plant \\
Yankee Atomic Electric Company & Kewaunee Nuclear Power Plant & 41.0 \\
& & 41.2
\end{tabular}





\section{UTILITY/COMPANY RELATIONSHIPS ORDERED BY PARENT/HOLDING COMPANY}



Table 3. Utility/Company Relationships by Parent/Holding Company

\begin{tabular}{|c|c|c|}
\hline Parent/Holding Company & Subsidiary & $\begin{array}{l}\text { Ownership } \\
\text { Percentage } \\
\end{array}$ \\
\hline Alliant Energy & $\begin{array}{l}\text { IES Utilities, Inc. } \\
\text { Interstate Power Company } \\
\text { Wisconsin Power \& Light Company }\end{array}$ & $\begin{array}{l}100.0 \\
100.0 \\
100.0\end{array}$ \\
\hline Ameren Corporation & AmerenUE & 100.0 \\
\hline American Electric Power Company & Indiana Michigan Power Company & 100.0 \\
\hline Atlantic Energy, Inc. & Atlantic City Electric Company & 100.0 \\
\hline Bangor Hydro-Electric Company & Maine Yankee Atomic Power Company & 7.0 \\
\hline BayCorp Holdings, Ltd. & $\begin{array}{l}\text { Great Bay Power Corporation } \\
\text { Little Bay Power Corporation }\end{array}$ & $\begin{array}{l}100.0 \\
100.0\end{array}$ \\
\hline Boston Edison Company & $\begin{array}{l}\text { Connecticut Yankee Atomic Power Company } \\
\text { Yankee Atomic Electric Company }\end{array}$ & $\begin{array}{l}9.5 \\
9.5\end{array}$ \\
\hline British Energy & AmerGen Energy Company & 50.0 \\
\hline Burlington Electric Department & Vermont Yankee Nuclear Power Corporation & 3.6 \\
\hline Cambridge Electric Light Company & $\begin{array}{l}\text { Maine Yankee Atomic Power Company } \\
\text { Vermont Yankee Nuclear Power Corporation } \\
\text { Yankee Atomic Electric Company }\end{array}$ & $\begin{array}{l}4.0 \\
2.5 \\
2.0\end{array}$ \\
\hline Central and South West Corporation & Central Power \& Light & 100.0 \\
\hline Central Maine Power Company & $\begin{array}{l}\text { Connecticut Yankee Atomic Power Company } \\
\text { Maine Yankee Atomic Power Company } \\
\text { Vermont Yankee Nuclear Power Corporation } \\
\text { Yankee Atomic Electric Company }\end{array}$ & $\begin{array}{r}6.0 \\
38.0 \\
4.0 \\
9.5\end{array}$ \\
\hline Central Vermont Public Service Corporation & $\begin{array}{l}\text { Connecticut Yankee Atomic Power Company } \\
\text { Maine Yankee Atomic Power Company } \\
\text { Vermont Yankee Nuclear Power Corporation }\end{array}$ & $\begin{array}{r}2.0 \\
2.0 \\
31.3\end{array}$ \\
\hline
\end{tabular}


Table 3. Utility/Company Relationships by Parent/Holding Company

Parent/Holding Company

Central Vermont Public Service Corporation (continued)

CMS Energy Corporation

Commonwealth Electric Company

Commonwealth Energy System

Connecticut Light \& Power Company

$\stackrel{\omega}{N}$

Consolidated Edison, Inc.

Constellation Energy Group

Dominion Resources, Inc.

DTE Energy Company

Duke Energy Corporation

Eastern Utilities Associates

Edison International

Enron Corporation

Entergy Corporation
Subsidiary

Ownership

Percentage

Yankee Atomic Electric Company

Consumers Energy

100.0

Connecticut Yankee Atomic Power Company

4.5

Yankee Atomic Electric Company

2.5

$\begin{array}{ll}\text { Commonwealth Electric Company } & 100.0\end{array}$

Connecticut Yankee Atomic Power Company 34.5

Maine Yankee Atomic Power Company

12.0

Vermont Yankee Nuclear Power Corporation $\quad 9.5$

Yankee Atomic Electric Company

24.5

Consolidated Edison Company of New York 100.0

Baltimore Gas \& Electric Company $\quad 100.0$

Constellation Nuclear Services $\quad 100.0$

Dominion Generation $\quad 100.0$

Virginia Electric and Power Company $\quad 100.0$

$\begin{array}{ll}\text { Detroit Edison Company } & 100.0\end{array}$

$\begin{array}{ll}\text { Duke Power } & 100.0\end{array}$

$\begin{array}{ll}\text { Montaup Electric Company } & 100.0\end{array}$

$\begin{array}{ll}\text { Southern California Edison Company } & 100.0\end{array}$

$\begin{array}{ll}\text { Portland General Electric Company } & 100.0\end{array}$

Entergy Arkansas, Inc. $\quad 100.0$

Entergy Gulf States, Inc. $\quad 100.0$

Entergy Louisiana, Inc. $\quad 100.0$

Entergy Nuclear Generation Co. $\quad 100.0$ 
Table 3. Utility/Company Relationships by Parent/Holding Company

Parent/Holding Company

Entergy Corporation (continued)

FirstEnergy Corporation

Florida Progress Corporation

FPL Group, Inc.

GPU, Inc.

Green Mountain Power Corporation

Kansas City Power \& Light Company

Kansas Electric Power Cooperative, Inc.

Kansas Gas \& Electric Company

Lyndonville (Village of) Electric Department

Maine Public Service Company

Montaup Electric Company

New England Electric System
Subsidiary

Ownership

Percentage

Entergy Operations, Inc.

100.0

System Energy Resources, Inc.

100.0

Cleveland Electric Illuminating Company $\quad 100.0$

FirstEnergy Nuclear Operating Company 100.0

Ohio Edison Company

100.0

Toledo Edison Company

100.0

Florida Power Corporation

100.0

Florida Power \& Light Company

GPU Nuclear, Inc.

Jersey Central Power \& Light Company

Metropolitan Edison Company

Pennsylvania Electric Company

100.0

100.0

100.0

100.0

Vermont Yankee Nuclear Power Corporation

Wolf Creek Nuclear Operating Corporation

Wolf Creek Nuclear Operating Corporation

Wolf Creek Nuclear Operating Corporation

Vermont Yankee Nuclear Power Corporation

Maine Yankee Atomic Power Company

Connecticut Yankee Atomic Power Company

Maine Yankee Atomic Power Company

4.0

Vermont Yankee Nuclear Power Corporation

2.5

Yankee Atomic Electric Company

4.5

New England Power Company 
Table 3. Utility/Company Relationships by Parent/Holding Company

\begin{tabular}{|c|c|c|}
\hline Parent/Holding Company & Subsidiary & $\begin{array}{l}\text { Ownership } \\
\text { Percentage }\end{array}$ \\
\hline New England Power Company & $\begin{array}{l}\text { Connecticut Yankee Atomic Power Company } \\
\text { Maine Yankee Atomic Power Company } \\
\text { Vermont Yankee Nuclear Power Corporation } \\
\text { Yankee Atomic Electric Company }\end{array}$ & $\begin{array}{l}15.0 \\
20.0 \\
20.0 \\
30.0\end{array}$ \\
\hline Niagara Mohawk Holdings, Inc. & Niagara Mohawk Power Corporation & 100.0 \\
\hline Northeast Utilities & $\begin{array}{l}\text { Connecticut Light \& Power Company } \\
\text { North Atlantic Energy Corporation } \\
\text { North Atlantic Energy Service Corporation } \\
\text { Northeast Nuclear Energy Co. } \\
\text { Public Service Company of New Hampshire } \\
\text { Western Massachusetts Electric Company }\end{array}$ & $\begin{array}{l}100.0 \\
100.0 \\
100.0 \\
100.0 \\
100.0 \\
100.0\end{array}$ \\
\hline NSTAR & $\begin{array}{l}\text { Boston Edison Company } \\
\text { Cambridge Electric Light Company }\end{array}$ & $\begin{array}{l}100.0 \\
100.0\end{array}$ \\
\hline Ohio Edison Company & Pennsylvania Power Company & 100.0 \\
\hline PACIFICORP & Pacific Power \& Light Company & 100.0 \\
\hline PECO Energy Company & AmerGen Energy Company & 50.0 \\
\hline PG\&E Corporation & Pacific Gas \& Electric Company & 100.0 \\
\hline Pinnacle West Capital Corporation & Arizona Public Service Company & 100.0 \\
\hline PP\&L Resources, Inc. & PP\&L, Inc. & 100.0 \\
\hline Public Service Company of New Hampshire & $\begin{array}{l}\text { Connecticut Yankee Atomic Power Company } \\
\text { Maine Yankee Atomic Power Company } \\
\text { Vermont Yankee Nuclear Power Corporation } \\
\text { Yankee Atomic Electric Company }\end{array}$ & $\begin{array}{l}5.0 \\
5.0 \\
4.0 \\
7.0\end{array}$ \\
\hline Public Service Enterprise Group, Inc. & $\begin{array}{l}\text { PSEG Power LLC } \\
\text { Public Service Electric \& Gas Company }\end{array}$ & $\begin{array}{l}100.0 \\
100.0\end{array}$ \\
\hline
\end{tabular}


Table 3. Utility/Company Relationships by Parent/Holding Company

\begin{tabular}{|c|c|c|}
\hline Parent/Holding Company & Subsidiary & $\begin{array}{l}\text { Ownership } \\
\text { Percentage }\end{array}$ \\
\hline Reliant Energy & Reliant Energy HL\&P & 100.0 \\
\hline RGS Energy Services, Inc. & Rochester Gas \& Electric Corporation & 100.0 \\
\hline SCANA Corporation & South Carolina Electric \& Gas Company & 100.0 \\
\hline Sempra Energy & San Diego Gas \& Electric Company & 100.0 \\
\hline Southern Company (The) & $\begin{array}{l}\text { Alabama Power Company } \\
\text { Georgia Power Company } \\
\text { Southern Nuclear Operating Company (The) }\end{array}$ & $\begin{array}{l}100.0 \\
100.0 \\
100.0\end{array}$ \\
\hline TXU & TXU Electric and Gas & 100.0 \\
\hline Unicom Corporation & Commonwealth Edison Company & 100.0 \\
\hline United Illuminating Company & Connecticut Yankee Atomic Power Company & 9.5 \\
\hline UNITIL Corporation & Fitchburg Gas \& Electric Company & 100.0 \\
\hline Vermont Electric Generation \& Transmission Cooperative, Inc. & Vermont Yankee Nuclear Power Corporation & 1.0 \\
\hline Washington Electric Cooperative & Vermont Yankee Nuclear Power Corporation & 0.6 \\
\hline Western Massachusetts Electric Company & $\begin{array}{l}\text { Connecticut Yankee Atomic Power Company } \\
\text { Maine Yankee Atomic Power Company } \\
\text { Vermont Yankee Nuclear Power Corporation } \\
\text { Yankee Atomic Electric Company }\end{array}$ & $\begin{array}{l}9.5 \\
3.0 \\
2.5 \\
7.0\end{array}$ \\
\hline Western Resources, Inc. & Kansas Gas \& Electric Company & 100.0 \\
\hline Wisconsin Energy Corporation & Wisconsin Electric Power Company & 100.0 \\
\hline WPS Resources Corporation & Wisconsin Public Service Corporation & 100.0 \\
\hline
\end{tabular}





\section{UTILITY/COMPANY RELATIONSHIPS ORDERED BY SUBSIDIARY}



Table 4. Utility/Company Relationships by Subsidiary

\begin{tabular}{|c|c|c|}
\hline Subsidiary & Parent/Holding Company & $\begin{array}{l}\text { Ownership } \\
\text { Percentage }\end{array}$ \\
\hline Alabama Power Company & Southern Company (The) & 100.0 \\
\hline AmerenUE & Ameren Corporation & 100.0 \\
\hline AmerGen Energy Company & $\begin{array}{l}\text { British Energy } \\
\text { PECO Energy Company }\end{array}$ & $\begin{array}{l}50.0 \\
50.0\end{array}$ \\
\hline Arizona Public Service Company & Pinnacle West Capital Corporation & 100.0 \\
\hline Atlantic City Electric Company & Atlantic Energy, Inc. & 100.0 \\
\hline Baltimore Gas \& Electric Company & Constellation Energy Group & 100.0 \\
\hline Boston Edison Company & NSTAR & 100.0 \\
\hline Cambridge Electric Light Company & NSTAR & 100.0 \\
\hline Central Power \& Light & Central and South West Corporation & 100.0 \\
\hline Cleveland Electric Illuminating Company & FirstEnergy Corporation & 100.0 \\
\hline Commonwealth Edison Company & Unicom Corporation & 100.0 \\
\hline Commonwealth Electric Company & Commonwealth Energy System & 100.0 \\
\hline Connecticut Light \& Power Company & Northeast Utilities & 100.0 \\
\hline Connecticut Yankee Atomic Power Company & $\begin{array}{l}\text { Boston Edison Company } \\
\text { Central Maine Power Company } \\
\text { Central Vermont Public Service Corporation } \\
\text { Commonwealth Electric Company } \\
\text { Connecticut Light \& Power Company } \\
\text { Montaup Electric Company } \\
\text { New England Power Company } \\
\text { Public Service Company of New Hampshire } \\
\text { United Illuminating Company }\end{array}$ & $\begin{array}{r}9.5 \\
6.0 \\
2.0 \\
4.5 \\
34.5 \\
4.5 \\
15.0 \\
5.0 \\
9.5\end{array}$ \\
\hline
\end{tabular}


Table 4. Utility/Company Relationships by Subsidiary

\begin{tabular}{|c|c|c|}
\hline Subsidiary & Parent/Holding Company & $\begin{array}{l}\text { Ownership } \\
\text { Percentage }\end{array}$ \\
\hline Connecticut Yankee Atomic Power Company (continued) & Western Massachusetts Electric Company & 9.5 \\
\hline Consolidated Edison Company of New York & Consolidated Edison, Inc. & 100.0 \\
\hline Constellation Nuclear Services & Constellation Energy Group & 100.0 \\
\hline Consumers Energy & CMS Energy Corporation & 100.0 \\
\hline Detroit Edison Company & DTE Energy Company & 100.0 \\
\hline Dominion Generation & Dominion Resources, Inc. & 100.0 \\
\hline Duke Power & Duke Energy Corporation & 100.0 \\
\hline Entergy Arkansas, Inc. & Entergy Corporation & 100.0 \\
\hline Entergy Gulf States, Inc. & Entergy Corporation & 100.0 \\
\hline Entergy Louisiana, Inc. & Entergy Corporation & 100.0 \\
\hline Entergy Nuclear Generation Co. & Entergy Corporation & 100.0 \\
\hline Entergy Operations, Inc. & Entergy Corporation & 100.0 \\
\hline FirstEnergy Nuclear Operating Company & FirstEnergy Corporation & 100.0 \\
\hline Fitchburg Gas \& Electric Company & UNITIL Corporation & 100.0 \\
\hline Florida Power \& Light Company & FPL Group, Inc. & 100.0 \\
\hline Florida Power Corporation & Florida Progress Corporation & 100.0 \\
\hline Georgia Power Company & Southern Company (The) & 100.0 \\
\hline GPU Nuclear, Inc. & GPU, Inc. & 100.0 \\
\hline
\end{tabular}


Table 4. Utility/Company Relationships by Subsidiary

$\begin{array}{lll}\text { Subsidiary Ownership } & \text { Parent/Holding Company } \\ \text { Percentage }\end{array}$

Great Bay Power Corporation

IES Utilities, Inc.

Indiana Michigan Power Company

Interstate Power Company

Jersey Central Power \& Light Company

Kansas Gas \& Electric Company

Little Bay Power Corporation

Maine Yankee Atomic Power Company

Metropolitan Edison Company

Montaup Electric Company

New England Power Company

Niagara Mohawk Power Corporation

North Atlantic Energy Corporation

North Atlantic Energy Service Corporation
BayCorp Holdings, Ltd.

100.0

Alliant Energy

100.0

American Electric Power Company

Alliant Energy

GPU, Inc.

Western Resources, Inc.

100.0

BayCorp Holdings, Ltd.

100.0

Bangor Hydro-Electric Company

Cambridge Electric Light Company

Central Maine Power Company

Central Vermont Public Service Corporation

Connecticut Light \& Power Company

Maine Public Service Company

Montaup Electric Company

New England Power Company

Public Service Company of New Hampshire

Western Massachusetts Electric Company

4.0

38.0

2.0

12.0

5.0

4.0

20.0

5.0

3.0

GPU, Inc.

100.0

Eastern Utilities Associates

100.0

New England Electric System

100.0

Niagara Mohawk Holdings, Inc.

100.0

Northeast Utilities

100.0

Northeast Utilities 
Table 4. Utility/Company Relationships by Subsidiary

\begin{tabular}{|c|c|c|}
\hline Subsidiary & Parent/Holding Company & $\begin{array}{l}\text { Ownership } \\
\text { Percentage }\end{array}$ \\
\hline Northeast Nuclear Energy Co. & Northeast Utilities & 100.0 \\
\hline Ohio Edison Company & FirstEnergy Corporation & 100.0 \\
\hline Pacific Gas \& Electric Company & PG\&E Corporation & 100.0 \\
\hline Pacific Power \& Light Company & PACIFICORP & 100.0 \\
\hline Pennsylvania Electric Company & GPU, Inc. & 100.0 \\
\hline Pennsylvania Power Company & Ohio Edison Company & 100.0 \\
\hline Portland General Electric Company & Enron Corporation & 100.0 \\
\hline PP\&L, Inc. & PP\&L Resources, Inc. & 100.0 \\
\hline PSEG Power LLC & Public Service Enterprise Group, Inc. & 100.0 \\
\hline Public Service Company of New Hampshire & Northeast Utilities & 100.0 \\
\hline Public Service Electric \& Gas Company & Public Service Enterprise Group, Inc. & 100.0 \\
\hline Reliant Energy HL\&P & Reliant Energy & 100.0 \\
\hline Rochester Gas \& Electric Corporation & RGS Energy Services, Inc. & 100.0 \\
\hline San Diego Gas \& Electric Company & Sempra Energy & 100.0 \\
\hline South Carolina Electric \& Gas Company & SCANA Corporation & 100.0 \\
\hline Southern California Edison Company & Edison International & 100.0 \\
\hline Southern Nuclear Operating Company (The) & Southern Company (The) & 100.0 \\
\hline System Energy Resources, Inc. & Entergy Corporation & 100.0 \\
\hline
\end{tabular}


Table 4. Utility/Company Relationships by Subsidiary

\begin{tabular}{|c|c|c|}
\hline Subsidiary & Parent/Holding Company & $\begin{array}{l}\text { Ownership } \\
\text { Percentage }\end{array}$ \\
\hline Toledo Edison Company & FirstEnergy Corporation & 100.0 \\
\hline TXU Electric and Gas & TXU & 100.0 \\
\hline \multirow[t]{13}{*}{ Vermont Yankee Nuclear Power Corporation } & Burlington Electric Department & 3.6 \\
\hline & Cambridge Electric Light Company & 2.5 \\
\hline & Central Maine Power Company & 4.0 \\
\hline & Central Vermont Public Service Corporation & 31.3 \\
\hline & Connecticut Light \& Power Company & 9.5 \\
\hline & Green Mountain Power Corporation & 17.90 \\
\hline & Lyndonville (Village of) Electric Department & 0.6 \\
\hline & Montaup Electric Company & 2.5 \\
\hline & New England Power Company & 20.0 \\
\hline & Public Service Company of New Hampshire & 4.0 \\
\hline & Vermont Electric Generation \& Transmission Cooperative, Inc. & 1.0 \\
\hline & Washington Electric Cooperative & 0.6 \\
\hline & Western Massachusetts Electric Company & 2.5 \\
\hline Virginia Electric and Power Company & Dominion Resources, Inc. & 100.0 \\
\hline Western Massachusetts Electric Company & Northeast Utilities & 100.0 \\
\hline Wisconsin Electric Power Company & Wisconsin Energy Corporation & 100.0 \\
\hline Wisconsin Power \& Light Company & Alliant Energy & 100.0 \\
\hline Wisconsin Public Service Corporation & WPS Resources Corporation & 100.0 \\
\hline \multirow[t]{3}{*}{ Wolf Creek Nuclear Operating Corporation } & Kansas City Power \& Light Company & 47.0 \\
\hline & Kansas Electric Power Cooperative, Inc. & 6.0 \\
\hline & Kansas Gas \& Electric Company & 47.0 \\
\hline \multirow[t]{4}{*}{ Yankee Atomic Electric Company } & Boston Edison Company & 9.5 \\
\hline & Cambridge Electric Light Company & 2.0 \\
\hline & Central Maine Power Company & 9.5 \\
\hline & Central Vermont Public Service Corporation & 3.5 \\
\hline
\end{tabular}


Table 4. Utility/Company Relationships by Subsidiary

\begin{tabular}{|c|c|c|}
\hline Subsidiary & Parent/Holding Company & $\begin{array}{l}\text { Ownership } \\
\text { Percentage }\end{array}$ \\
\hline \multirow[t]{6}{*}{ Yankee Atomic Electric Company (continued) } & Commonwealth Electric Company & 2.5 \\
\hline & Connecticut Light \& Power Company & 24.5 \\
\hline & Montaup Electric Company & 4.5 \\
\hline & New England Power Company & 30.0 \\
\hline & Public Service Company of New Hampshire & 7.0 \\
\hline & Western Massachusetts Electric Company & 7.0 \\
\hline
\end{tabular}




\section{NUCLEAR POWER PLANTS LISTED BY OPERATOR}





\begin{tabular}{|c|c|}
\hline Operator & Plant Name \\
\hline AmerenUE & Callaway Plant \\
\hline \multirow[t]{2}{*}{ AmerGen Energy Company } & Clinton Power Station \\
\hline & Three Mile Island Nuclear Station, Unit 1 \\
\hline Arizona Public Service Company & Palo Verde Nuclear Generating Station, Units 1-3 \\
\hline Baltimore Gas \& Electric Company & Calvert Cliffs Nuclear Power Plant, Units 1 \& 2 \\
\hline \multirow[t]{3}{*}{ Carolina Power \& Light Company } & Brunswick Steam Electric Plant, Units 1 \& 2 \\
\hline & Harris (Shearon) Nuclear Power Plant \\
\hline & Robinson (H. B.) Plant, Unit 2 \\
\hline \multirow[t]{7}{*}{ Commonwealth Edison Company } & Braidwood Station, Units $1 \& 2$ \\
\hline & Byron Station, Units $1 \& 2$ \\
\hline & Dresden Nuclear Power Station, Unit 1 \\
\hline & Dresden Nuclear Power Station, Units 2 \& 3 \\
\hline & LaSalle County Station, Units 1 \& 2 \\
\hline & Quad Cities Station, Units $1 \& 2$ \\
\hline & Zion Nuclear Plant, Units 1 \& 2 \\
\hline \multirow[t]{2}{*}{ Consolidated Edison Company of New York } & Indian Point, Unit 1 \\
\hline & Indian Point, Unit 2 \\
\hline \multirow[t]{2}{*}{ Consumers Energy } & Big Rock Point Nuclear Plant \\
\hline & Palisades Nuclear Plant \\
\hline Dairyland Power Cooperative & LaCrosse (Genoa) Nuclear Generating Station \\
\hline Detroit Edison Company & Fermi (Enrico) Atomic Power Plant, Unit 2 \\
\hline \multirow[t]{2}{*}{ Dominion Generation } & North Anna Power Station, Units $1 \& 2$ \\
\hline & Surry Power Station, Units $1 \& 2$ \\
\hline \multirow[t]{4}{*}{ Duke Power } & Catawba Nuclear Station, Unit 1 \\
\hline & Catawba Nuclear Station, Unit 2 \\
\hline & McGuire (William B.) Nuclear Station, Units $1 \& 2$ \\
\hline & Oconee Nuclear Station, Units 1-3 \\
\hline \multirow[t]{3}{*}{ Energy Northwest } & WNP-2 \\
\hline & WPPSS Nuclear Project, Unit 1 \\
\hline & WPPSS Nuclear Project, Unit 3 \\
\hline
\end{tabular}


Table 5. Nuclear Power Plants Listed by Operator

\begin{tabular}{|c|c|}
\hline Operator & Plant Name \\
\hline Entergy Nuclear Generation Co. & Pilgrim Station \\
\hline Entergy Operations, Inc. & $\begin{array}{l}\text { Arkansas Nuclear One, Units } 1 \& 2 \\
\text { Grand Gulf Nuclear Station } \\
\text { River Bend Station } \\
\text { Waterford Generating Station, Unit } 3\end{array}$ \\
\hline FirstEnergy Nuclear Operating Company & $\begin{array}{l}\text { Beaver Valley Power Station, Unit } 1 \\
\text { Beaver Valley Power Station, Unit } 2 \\
\text { Davis-Besse Nuclear Power Station } \\
\text { Perry Nuclear Power Plant }\end{array}$ \\
\hline Florida Power \& Light Company & $\begin{array}{l}\text { St. Lucie Plant, Unit } 1 \\
\text { St. Lucie Plant, Unit } 2 \\
\text { Turkey Point Station, Unit } 3 \text { \& } 4\end{array}$ \\
\hline Florida Power Corporation & Crystal River Nuclear Plant, Unit 3 \\
\hline General Electric Company & Vallecitos Boiling Water Reactor \\
\hline GPU Nuclear, Inc. & $\begin{array}{l}\text { Oyster Creek Nuclear Power Plant } \\
\text { Three Mile Island Nuclear Station, Unit } 2\end{array}$ \\
\hline IES Utilities, Inc. & Arnold (Duane) Energy Center \\
\hline Indiana Michigan Power Company & Cook (Donald C.) Nuclear Power Plant, Units $1 \& 2$ \\
\hline Long Island Power Authority & Shoreham Nuclear Power Station \\
\hline Maine Yankee Atomic Power Company & Maine Yankee Atomic Power Station \\
\hline Nebraska Public Power District & Cooper Nuclear Station \\
\hline New Century Energies & Fort St. Vrain Nuclear Generating Station \\
\hline New York Power Authority & $\begin{array}{l}\text { Fitzpatrick (James A.) Nuclear Power Plant } \\
\text { Indian Point, Unit } 3\end{array}$ \\
\hline Niagara Mohawk Power Corporation & $\begin{array}{l}\text { Nine Mile Point Nuclear Station, Unit } 1 \\
\text { Nine Mile Point Nuclear Station, Unit } 2\end{array}$ \\
\hline North Atlantic Energy Service Corporation & Seabrook Nuclear Power Station \\
\hline Northeast Nuclear Energy Co. & Millstone Nuclear Power Station, Unit 2 \\
\hline
\end{tabular}

NUREG/CR-6500, Rev. 1 


\begin{tabular}{|c|c|}
\hline Operator & Plant Name \\
\hline Northeast Nuclear Energy Co. & Millstone Nuclear Power Station, Unit 3 \\
\hline Northeast Utilities & $\begin{array}{l}\text { Haddam Neck (Connecticut Yankee) Plant } \\
\text { Millstone Nuclear Power Station, Unit } 1\end{array}$ \\
\hline Northern States Power Company & $\begin{array}{l}\text { Monticello Nuclear Generating Plant } \\
\text { Pathfinder Atomic Plant } \\
\text { Prairie Island Nuclear Plant, Units } 1 \& 2\end{array}$ \\
\hline Omaha Public Power District & Fort Calhoun Station \\
\hline Pacific Gas \& Electric Company & $\begin{array}{l}\text { Diablo Canyon Nuclear Power Plant, Units } 1 \text { \& } 2 \\
\text { Humboldt Bay Power Plant, Unit } 3\end{array}$ \\
\hline PECO Energy Company & $\begin{array}{l}\text { Limerick Generating Station, Units } 1 \& 2 \\
\text { Peach Bottom Atomic Power Station, Unit } 1 \\
\text { Peach Bottom Atomic Power Station, Units } 2 \text { \& } 3\end{array}$ \\
\hline Portland General Electric Company & Trojan Nuclear Plant \\
\hline Power Reactor Development Company & Fermi (Enrico) Atomic Power Plant, Unit 1 \\
\hline PP\&L, Inc. & Susquehanna Steam Electric Station, Units 1 \& 2 \\
\hline Public Service Electric \& Gas Company & $\begin{array}{l}\text { Hope Creek Nuclear Generating Station } \\
\text { Salem Nuclear Generating Station, Units } 1 \& 2\end{array}$ \\
\hline Rochester Gas \& Electric Corporation & Ginna (Robert E.) Nuclear Power Plant \\
\hline Sacramento Municipal Utility District & Rancho Seco Nuclear Generating Station \\
\hline Saxton Nuclear Experimental Corporation & Saxton Nuclear Experimental Reactor Project \\
\hline South Carolina Electric \& Gas Company & Summer (Virgil C.) Nuclear Station \\
\hline Southern California Edison Company & $\begin{array}{l}\text { San Onofre Nuclear Generating Station, Unit } 1 \\
\text { San Onofre Nuclear Generating Station, Units } 2 \text { \& } 3\end{array}$ \\
\hline Southern Nuclear Operating Company (The) & $\begin{array}{l}\text { Farley (Joseph M.) Nuclear Plant, Units } 1 \& 2 \\
\text { Hatch (Edwin I.) Nuclear Plant, Units } 1 \& 2 \\
\text { Vogtle (Alvin W.) Nuclear Plant, Units } 1 \& 2\end{array}$ \\
\hline STP Nuclear Operating Company & South Texas Project, Units 1 \& 2 \\
\hline
\end{tabular}


Table 5. Nuclear Power Plants Listed by Operator

\begin{tabular}{ll}
\hline Operator & Plant Name \\
\hline Tennessee Valley Authority & $\begin{array}{l}\text { Bellefonte Nuclear Plant, Units } 1 \text { \& } 2 \\
\text { Browns Ferry Nuclear Power Station, Unit 1 } \\
\text { Browns Ferry Nuclear Power Station, Units 2 \& 3 } \\
\text { Sequoyah Nuclear Plant, Units 1 \& 2 } \\
\text { Watts Bar Nuclear Plant, Unit 1 } \\
\text { Watts Bar Nuclear Plant, Unit } 2\end{array}$ \\
TXU Electric and Gas & Comanche Peak Steam Electric Station, Units 1 \& 2 \\
Vermont Yankee Nuclear Power Corporation & Vermont Yankee Nuclear Power Station \\
Wisconsin Electric Power Company & Point Beach Nuclear Plant, Units 1 \& 2 \\
Wisconsin Public Service Corporation & Kewaunee Nuclear Power Plant \\
Wolf Creek Nuclear Operating Corporation & Wolf Creek Generating Station \\
Yankee Atomic Electric Company & Yankee Nuclear Power Station
\end{tabular}




\section{NUCLEAR POWER PLANT OPERATORS LISTED BY PLANT NAME}



Table 6. Nuclear Power Plant Operators Listed by Plant

\begin{tabular}{|c|c|}
\hline Plant Name & Operator \\
\hline Arkansas Nuclear One, Units 1 \& 2 & Entergy Operations, Inc. \\
\hline Arnold (Duane) Energy Center & IES Utilities, Inc. \\
\hline Beaver Valley Power Station, Unit 1 & FirstEnergy Nuclear Operating Company \\
\hline Beaver Valley Power Station, Unit 2 & FirstEnergy Nuclear Operating Company \\
\hline Bellefonte Nuclear Plant, Units $1 \& 2$ & Tennessee Valley Authority \\
\hline Big Rock Point Nuclear Plant & Consumers Energy \\
\hline Braidwood Station, Units $1 \& 2$ & Commonwealth Edison Company \\
\hline Browns Ferry Nuclear Power Station, Unit 1 & Tennessee Valley Authority \\
\hline Browns Ferry Nuclear Power Station, Units 2 \& 3 & Tennessee Valley Authority \\
\hline Brunswick Steam Electric Plant, Units 1 \& 2 & Carolina Power \& Light Company \\
\hline Byron Station, Units $1 \& 2$ & Commonwealth Edison Company \\
\hline Callaway Plant & AmerenUE \\
\hline Calvert Cliffs Nuclear Power Plant, Units 1 \& 2 & Baltimore Gas \& Electric Company \\
\hline Catawba Nuclear Station, Unit 1 & Duke Power \\
\hline Catawba Nuclear Station, Unit 2 & Duke Power \\
\hline Clinton Power Station & AmerGen Energy Company \\
\hline Comanche Peak Steam Electric Station, Units 1 \& 2 & TXU Electric and Gas \\
\hline Cook (Donald C.) Nuclear Power Plant, Units 1 \& 2 & Indiana Michigan Power Company \\
\hline Cooper Nuclear Station & Nebraska Public Power District \\
\hline Crystal River Nuclear Plant, Unit 3 & Florida Power Corporation \\
\hline Davis-Besse Nuclear Power Station & FirstEnergy Nuclear Operating Company \\
\hline Diablo Canyon Nuclear Power Plant, Units 1 \& 2 & Pacific Gas \& Electric Company \\
\hline Dresden Nuclear Power Station, Unit 1 & Commonwealth Edison Company \\
\hline Dresden Nuclear Power Station, Units 2 \& 3 & Commonwealth Edison Company \\
\hline Farley (Joseph M.) Nuclear Plant, Units 1 \& 2 & Southern Nuclear Operating Company (The) \\
\hline Fermi (Enrico) Atomic Power Plant, Unit 1 & Power Reactor Development Company \\
\hline Fermi (Enrico) Atomic Power Plant, Unit 2 & Detroit Edison Company \\
\hline Fitzpatrick (James A.) Nuclear Power Plant & New York Power Authority \\
\hline Fort Calhoun Station & Omaha Public Power District \\
\hline Fort St. Vrain Nuclear Generating Station & New Century Energies \\
\hline Ginna (Robert E.) Nuclear Power Plant & Rochester Gas \& Electric Corporation \\
\hline Grand Gulf Nuclear Station & Entergy Operations, Inc. \\
\hline Haddam Neck (Connecticut Yankee) Plant & Northeast Utilities \\
\hline Harris (Shearon) Nuclear Power Plant & Carolina Power \& Light Company \\
\hline Hatch (Edwin I.) Nuclear Plant, Units 1 \& 2 & Southern Nuclear Operating Company (The) \\
\hline Hope Creek Nuclear Generating Station & Public Service Electric \& Gas Company \\
\hline Humboldt Bay Power Plant, Unit 3 & Pacific Gas \& Electric Company \\
\hline Indian Point, Unit 1 & Consolidated Edison Company of New York \\
\hline
\end{tabular}


Table 6. Nuclear Power Plant Operators Listed by Plant

\begin{tabular}{|c|c|}
\hline Plant Name & Operator \\
\hline Indian Point, Unit 2 & Consolidated Edison Company of New York \\
\hline Indian Point, Unit 3 & New York Power Authority \\
\hline Kewaunee Nuclear Power Plant & Wisconsin Public Service Corporation \\
\hline LaCrosse (Genoa) Nuclear Generating Station & Dairyland Power Cooperative \\
\hline LaSalle County Station, Units $1 \& 2$ & Commonwealth Edison Company \\
\hline Limerick Generating Station, Units $1 \& 2$ & PECO Energy Company \\
\hline Maine Yankee Atomic Power Station & Maine Yankee Atomic Power Company \\
\hline McGuire (William B.) Nuclear Station, Units 1 \& 2 & Duke Power \\
\hline Millstone Nuclear Power Station, Unit 1 & Northeast Utilities \\
\hline Millstone Nuclear Power Station, Unit 2 & Northeast Nuclear Energy Co. \\
\hline Millstone Nuclear Power Station, Unit 3 & Northeast Nuclear Energy Co. \\
\hline Monticello Nuclear Generating Plant & Northern States Power Company \\
\hline Nine Mile Point Nuclear Station, Unit 1 & Niagara Mohawk Power Corporation \\
\hline Nine Mile Point Nuclear Station, Unit 2 & Niagara Mohawk Power Corporation \\
\hline North Anna Power Station, Units $1 \& 2$ & Dominion Generation \\
\hline Oconee Nuclear Station, Units 1-3 & Duke Power \\
\hline Oyster Creek Nuclear Power Plant & GPU Nuclear, Inc. \\
\hline Palisades Nuclear Plant & Consumers Energy \\
\hline Palo Verde Nuclear Generating Station, Units 1-3 & Arizona Public Service Company \\
\hline Pathfinder Atomic Plant & Northern States Power Company \\
\hline Peach Bottom Atomic Power Station, Unit 1 & PECO Energy Company \\
\hline Peach Bottom Atomic Power Station, Units 2 \& 3 & PECO Energy Company \\
\hline Perry Nuclear Power Plant & FirstEnergy Nuclear Operating Company \\
\hline Pilgrim Station & Entergy Nuclear Generation Co. \\
\hline Point Beach Nuclear Plant, Units 1 \& 2 & Wisconsin Electric Power Company \\
\hline Prairie Island Nuclear Plant, Units $1 \& 2$ & Northern States Power Company \\
\hline Quad Cities Station, Units $1 \& 2$ & Commonwealth Edison Company \\
\hline Rancho Seco Nuclear Generating Station & Sacramento Municipal Utility District \\
\hline River Bend Station & Entergy Operations, Inc. \\
\hline Robinson (H. B.) Plant, Unit 2 & Carolina Power \& Light Company \\
\hline Salem Nuclear Generating Station, Units 1 \& 2 & Public Service Electric \& Gas Company \\
\hline San Onofre Nuclear Generating Station, Unit 1 & Southern California Edison Company \\
\hline San Onofre Nuclear Generating Station, Units 2 \& 3 & Southern California Edison Company \\
\hline Saxton Nuclear Experimental Reactor Project & Saxton Nuclear Experimental Corporation \\
\hline
\end{tabular}

NUREG/CR-6500, Rev. 1 
Table 6. Nuclear Power Plant Operators Listed by Plant

\begin{tabular}{|c|c|}
\hline Plant Name & Operator \\
\hline Seabrook Nuclear Power Station & North Atlantic Energy Service Corporation \\
\hline Sequoyah Nuclear Plant, Units $1 \& 2$ & Tennessee Valley Authority \\
\hline Shoreham Nuclear Power Station & Long Island Power Authority \\
\hline South Texas Project, Units $1 \& 2$ & STP Nuclear Operating Company \\
\hline St. Lucie Plant, Unit 1 & Florida Power \& Light Company \\
\hline St. Lucie Plant, Unit 2 & Florida Power \& Light Company \\
\hline Summer (Virgil C.) Nuclear Station & South Carolina Electric \& Gas Company \\
\hline Surry Power Station, Units $1 \& 2$ & Dominion Generation \\
\hline Susquehanna Steam Electric Station, Units 1 \& 2 & PP\&L, Inc. \\
\hline Three Mile Island Nuclear Station, Unit 1 & AmerGen Energy Company \\
\hline Three Mile Island Nuclear Station, Unit 2 & GPU Nuclear, Inc. \\
\hline Trojan Nuclear Plant & Portland General Electric Company \\
\hline Turkey Point Station, Unit $3 \& 4$ & Florida Power \& Light Company \\
\hline Vallecitos Boiling Water Reactor & General Electric Company \\
\hline Vermont Yankee Nuclear Power Station & Vermont Yankee Nuclear Power Corporation \\
\hline Vogtle (Alvin W.) Nuclear Plant, Units 1 \& 2 & Southern Nuclear Operating Company (The) \\
\hline Waterford Generating Station, Unit 3 & Entergy Operations, Inc. \\
\hline Watts Bar Nuclear Plant, Unit 1 & Tennessee Valley Authority \\
\hline Watts Bar Nuclear Plant, Unit 2 & Tennessee Valley Authority \\
\hline WNP-2 & Energy Northwest \\
\hline Wolf Creek Generating Station & Wolf Creek Nuclear Operating Corporation \\
\hline WPPSS Nuclear Project, Unit 1 & Energy Northwest \\
\hline WPPSS Nuclear Project, Unit 3 & Energy Northwest \\
\hline Yankee Nuclear Power Station & Yankee Atomic Electric Company \\
\hline Zion Nuclear Plant, Units 1 \& 2 & Commonwealth Edison Company \\
\hline
\end{tabular}





\section{NUCLEAR POWER PLANTS LISTED BY STATE}



Table 7. Nuclear Power Plants by State

\begin{tabular}{|c|c|}
\hline State & Plant Name \\
\hline Alabama & $\begin{array}{l}\text { Bellefonte Nuclear Plant, Units } 1 \& 2 \\
\text { Browns Ferry Nuclear Power Station, Unit } 1 \\
\text { Browns Ferry Nuclear Power Station, Units } 2 \text { \& } 3 \\
\text { Farley (Joseph M.) Nuclear Plant, Units } 1 \text { \& } 2\end{array}$ \\
\hline Arizona & Palo Verde Nuclear Generating Station, Units 1-3 \\
\hline Arkansas & Arkansas Nuclear One, Units $1 \& 2$ \\
\hline California & $\begin{array}{l}\text { Diablo Canyon Nuclear Power Plant, Units } 1 \text { \& } 2 \\
\text { Humboldt Bay Power Plant, Unit } 3 \\
\text { Rancho Seco Nuclear Generating Station } \\
\text { San Onofre Nuclear Generating Station, Unit } 1 \\
\text { San Onofre Nuclear Generating Station, Units } 2 \text { \& } 3 \\
\text { Vallecitos Boiling Water Reactor }\end{array}$ \\
\hline Colorado & Fort St. Vrain Nuclear Generating Station \\
\hline Connecticut & $\begin{array}{l}\text { Haddam Neck (Connecticut Yankee) Plant } \\
\text { Millstone Nuclear Power Station, Unit } 1 \\
\text { Millstone Nuclear Power Station, Unit } 2 \\
\text { Millstone Nuclear Power Station, Unit } 3\end{array}$ \\
\hline Florida & $\begin{array}{l}\text { Crystal River Nuclear Plant, Unit } 3 \\
\text { St. Lucie Plant, Unit } 1 \\
\text { St. Lucie Plant, Unit } 2 \\
\text { Turkey Point Station, Unit } 3 \text { \& } 4\end{array}$ \\
\hline Georgia & $\begin{array}{l}\text { Hatch (Edwin I.) Nuclear Plant, Units } 1 \& 2 \\
\text { Vogtle (Alvin W.) Nuclear Plant, Units } 1 \& 2\end{array}$ \\
\hline Illinois & $\begin{array}{l}\text { Braidwood Station, Units } 1 \text { \& } 2 \\
\text { Byron Station, Units } 1 \text { \& } 2 \\
\text { Clinton Power Station } \\
\text { Dresden Nuclear Power Station, Unit } 1 \\
\text { Dresden Nuclear Power Station, Units } 2 \& 3 \\
\text { LaSalle County Station, Units } 1 \text { \& } 2 \\
\text { Quad Cities Station, Units } 1 \& 2 \\
\text { Zion Nuclear Plant, Units } 1 \& 2\end{array}$ \\
\hline lowa & Arnold (Duane) Energy Center \\
\hline Kansas & Wolf Creek Generating Station \\
\hline
\end{tabular}


Table 7. Nuclear Power Plants by State

\begin{tabular}{|c|c|}
\hline State & Plant Name \\
\hline Louisiana & $\begin{array}{l}\text { River Bend Station } \\
\text { Waterford Generating Station, Unit } 3\end{array}$ \\
\hline Maine & Maine Yankee Atomic Power Station \\
\hline Maryland & Calvert Cliffs Nuclear Power Plant, Units 1 \& 2 \\
\hline Massachusetts & $\begin{array}{l}\text { Pilgrim Station } \\
\text { Yankee Nuclear Power Station }\end{array}$ \\
\hline Michigan & $\begin{array}{l}\text { Big Rock Point Nuclear Plant } \\
\text { Cook (Donald C.) Nuclear Power Plant, Units } 1 \& 2 \\
\text { Fermi (Enrico) Atomic Power Plant, Unit } 1 \\
\text { Fermi (Enrico) Atomic Power Plant, Unit } 2 \\
\text { Palisades Nuclear Plant }\end{array}$ \\
\hline Minnesota & $\begin{array}{l}\text { Monticello Nuclear Generating Plant } \\
\text { Prairie Island Nuclear Plant, Units } 1 \& 2\end{array}$ \\
\hline Mississippi & Grand Gulf Nuclear Station \\
\hline Missouri & Callaway Plant \\
\hline Nebraska & $\begin{array}{l}\text { Cooper Nuclear Station } \\
\text { Fort Calhoun Station }\end{array}$ \\
\hline New Hampshire & Seabrook Nuclear Power Station \\
\hline New Jersey & $\begin{array}{l}\text { Hope Creek Nuclear Generating Station } \\
\text { Oyster Creek Nuclear Power Plant } \\
\text { Salem Nuclear Generating Station, Units } 1 \& 2\end{array}$ \\
\hline New York & $\begin{array}{l}\text { Fitzpatrick (James A.) Nuclear Power Plant } \\
\text { Ginna (Robert E.) Nuclear Power Plant } \\
\text { Indian Point, Unit } 1 \\
\text { Indian Point, Unit } 2 \\
\text { Indian Point, Unit } 3 \\
\text { Nine Mile Point Nuclear Station, Unit } 1 \\
\text { Nine Mile Point Nuclear Station, Unit } 2 \\
\text { Shoreham Nuclear Power Station }\end{array}$ \\
\hline North Carolina & $\begin{array}{l}\text { Brunswick Steam Electric Plant, Units } 1 \& 2 \\
\text { Harris (Shearon) Nuclear Power Plant } \\
\text { McGuire (William B.) Nuclear Station, Units } 1 \& 2\end{array}$ \\
\hline
\end{tabular}


Table 7. Nuclear Power Plants by State

\begin{tabular}{|c|c|}
\hline State & Plant Name \\
\hline Ohio & $\begin{array}{l}\text { Davis-Besse Nuclear Power Station } \\
\text { Perry Nuclear Power Plant }\end{array}$ \\
\hline Oregon & Trojan Nuclear Plant \\
\hline Pennsylvania & $\begin{array}{l}\text { Beaver Valley Power Station, Unit } 1 \\
\text { Beaver Valley Power Station, Unit } 2 \\
\text { Limerick Generating Station, Units } 1 \text { \& } 2 \\
\text { Peach Bottom Atomic Power Station, Unit } 1 \\
\text { Peach Bottom Atomic Power Station, Units } 2 \text { \& } 3 \\
\text { Saxton Nuclear Experimental Reactor Project } \\
\text { Susquehanna Steam Electric Station, Units } 1 \text { \& } 2 \\
\text { Three Mile Island Nuclear Station, Unit } 1 \\
\text { Three Mile Island Nuclear Station, Unit } 2\end{array}$ \\
\hline South Carolina & $\begin{array}{l}\text { Catawba Nuclear Station, Unit } 1 \\
\text { Catawba Nuclear Station, Unit } 2 \\
\text { Oconee Nuclear Station, Units 1-3 } \\
\text { Robinson (H. B.) Plant, Unit } 2 \\
\text { Summer (Virgil C.) Nuclear Station }\end{array}$ \\
\hline South Dakota & Pathfinder Atomic Plant \\
\hline Tennessee & $\begin{array}{l}\text { Sequoyah Nuclear Plant, Units } 1 \& 2 \\
\text { Watts Bar Nuclear Plant, Unit } 1 \\
\text { Watts Bar Nuclear Plant, Unit } 2\end{array}$ \\
\hline Texas & $\begin{array}{l}\text { Comanche Peak Steam Electric Station, Units } 1 \& 2 \\
\text { South Texas Project, Units } 1 \& 2\end{array}$ \\
\hline Vermont & Vermont Yankee Nuclear Power Station \\
\hline Virginia & $\begin{array}{l}\text { North Anna Power Station, Units } 1 \& 2 \\
\text { Surry Power Station, Units } 1 \& 2\end{array}$ \\
\hline Washington & $\begin{array}{l}\text { WNP-2 } \\
\text { WPPSS Nuclear Project, Unit } 1 \\
\text { WPPSS Nuclear Project, Unit } 3\end{array}$ \\
\hline Wisconsin & $\begin{array}{l}\text { Kewaunee Nuclear Power Plant } \\
\text { LaCrosse (Genoa) Nuclear Generating Station } \\
\text { Point Beach Nuclear Plant, Units } 1 \& 2\end{array}$ \\
\hline
\end{tabular}





\section{OWNERSHIP NOTES}



Table 8. Ownership Notes

Utility

Alliant Energy

AmerenUE

AmerGen Energy Company

American Electric Power Company

Arizona Public Service Company

\section{Avista Utilities}

Carolina Power \& Light Company

Central and South West Corporation
Comments

Formerly Interstate Energy Corporation. In the process of forming a nuclear management company with Wisconsin Electric Power Company, Wisconsin Public Service Corporation, and Northern States Power.

\section{Formerly Union Electric Company.}

In the process of purchasing Nine Mile Point Unit 1 and $41 \%$ of Unit 2 from Niagara Mohawk Power Corporation; and 18\% of Nine Mile Point Unit 2 from New York State Electric \& Gas Corporation. Buying Vermont Yankee; deal expected to close July 2000. Buying Oyster Creek from GPU and will operate plant until license expires in 2009.

Merger with Central \& South West Corporation in process.

Will eventually have to transfer all of its generating assets to the newly formed Pinnacle West Energy.

Formerly Washington Water Power Company.

Acquisition of Florida Progress Corporation in process; will obtain Crystal River license pending NRC approval. Also plans to create a holding company for CP\&L in process; formation of holding company will involve indirect transfer of operating licenses for Brunswick Units 1 and 2, Harris, and Robinson. Acquisition of Florida Progress Corporation is not part of application on forming a holding company.

Merger with American Electric Power Company in process.

In process of selling ownership interest in Peach Bottom to Public Service Enterprise Group (PSEG) and PECO Energy Company; in Salem and Hope Creek to PSEG Power LLC.

Merger with Northeast Utilities in process.

Formerly Consumers Power Company.

Merger with MCN Energy Group in process; deal expected to close spring 2000.

Merger with New England Electric System in process (expected to be complete in early 2000). 


\section{Utility}

Energy Northwest

Entergy Corporation

Fitchburg Gas \& Electric Company

Florida Progress Corporation

GPU Nuclear, Inc.

Kansas City Power \& Light Company

MidAmerican Energy Holdings Company

จ New Century Energies

New England Electric System

New York Power Authority

New York State Electric \& Gas Corporation

Niagara Mohawk Power Corporation

Northeast Utilities

Northern States Power Company

PECO Energy Company

\section{Comments}

Formerly Washington Public Power Supply System.

Has entered exclusive negotiations for the potential purchase of New York Power Authority's FitzPatrick and Indian Point Unit 3 nuclear plants.

In process of selling its minority interest (about $0.2 \%$ ) of Millstone 3 to Select Energy.

Being purchased by Carolina Power \& Light Company.

Selling Oyster Creek to AmerGen; sale anticipated to be completed in spring 2000.

Merger with Western Resources in process; new company will be Westar Energy.

Formerly MidAmerican Energy Company.

Merger in process with Northern States Power Company to form Xcel Energy, Inc.

Merger with Eastern Utilities Associates in process (expected to be complete in early 2000). NEES is also in the process of merging with National Grid Group plc of Coventry, England.

Entergy Corporation has entered exclusive negotiations for the potential purchase of FitzPatrick and Indian Point Unit 3 nuclear plants.

In the process of selling $18 \%$ of Nine Mile Point Unit 2 to AmerGen.

In the process of selling Nine Mile Point Unit 1 and $41 \%$ of Unit 2 to AmerGen.

Merger with Consolidated Edison, Inc. in progress. Plans to auction the Millstone Station assets of its subsidiaries beginning November 1999. Subsidiaries' shares of Seabrook also being auctioned.

In the process of forming a nuclear management company with Alliant Energy, Wisconsin Public Service Corporation, and Wisconsin Electric Power Company. Merger in process with New Century Energies to form Xcel Energy Inc.

Merger with Unicom, owner of Commonwealth Edison, in process. In process of buying Conectiv's interest in Peach Bottom; deal expected to close mid-2000. 
Table 8. Ownership Notes

\begin{tabular}{ll}
\hline Utility Comments \\
\hline
\end{tabular}

Pinnacle West Energy

PP\&L, Inc.

PSEG Power LLC

Public Service Enterprise Group, Inc.

Reliant Energy

Reliant Energy HL\&P

San Diego Gas \& Electric Company

TXU

TXU Electric and Gas

Unicom Corporation

Vermont Yankee Nuclear Power Corporation

Western Resources, Inc.

Wisconsin Electric Power Company

Wisconsin Energy Corporation

Wisconsin Public Service Corporation
Will eventually acquire Arizona Public Service's generating assets.

Formerly Pennsylvania Power \& Light Company.

Unregulated power subsidiary of Public Service Enterprise Group, Inc. (PSEG). In process of buying $14.8 \%$ interest in Salem and $5 \%$ interest in Hope Creek from Conectiv; deal expected to close mid-2000.

In process of buying Conectiv's interest in Peach Bottom.

Formerly Houston Industries, Inc.

Formerly Houston Lighting \& Power.

Plans to auction its $20 \%$ share in San Onofre Nuclear Plant.

Formerly Texas Utilities Company.

Formerly TU Electric Company.

Merger with PECO in process.

In process of selling Vermont Yankee to AmerGen.

Merger with Kansas City Power \& Light Company in process. New company will be Westar Energy. In the process of forming a nuclear management company with Alliant Energy, Wisconsin Public Service Corporation, and Northern States Power.

Merger with WICOR, Inc. in process.

In the process of forming a nuclear management company with Alliant Energy, Wisconsin Electric Power Company, and Northern States Power. 



\section{NUCLEAR POWER PLANT OPERATOR WEB SITES}



Table 9. Nuclear Power Plant Operator Web Sites

\begin{tabular}{|c|c|}
\hline Utility & Web Site \\
\hline Alliant Energy & http://www.alliantenergy.com/index.html \\
\hline American Electric Power Company & http://www.aep.com/ \\
\hline AmerenUE & http://www.ameren.com/ameren2/index.htm \\
\hline Arizona Public Service Company & http://about.apsc.com/homepg/default.asp \\
\hline Baltimore Gas and Electric Company & http://www.bge.com/ \\
\hline Boston Edison Company & http://www.bedison.com/index2.htm \\
\hline Carolina Power \& Light Company & http://www.cplc.com/ \\
\hline Central lowa Power Cooperative & http://www.cipco.org/ \\
\hline Commonwealth Edison Company & http://www.ucm.com/homepage/homepage.asp \\
\hline Consolidated Edison Company of New York & http://www.coned.com/ \\
\hline Consumers Energy & http://www.consumersenergy.com/ \\
\hline Dairyland Power Cooperative & http://www.dairynet.com/index.html \\
\hline Detroit Edison Company & http://www.detroitedison.com/ \\
\hline Dominion Generation & http://www.domres.com/homepage.html \\
\hline Duke Power (Business Unit) & http://www.duke-energy.com/ \\
\hline Energy Northwest & http://www.wnp2.com/ \\
\hline Entergy Corporation & http://www.entergy.com/ \\
\hline Entergy Nuclear Generation Company & http://www.entergy.com/nuclear/ \\
\hline Florida Power Corporation & http://www.fpc.com/flpower/ \\
\hline Florida Power and Light Company & http://www.fpl.com/ \\
\hline FirstEnergy Nuclear Operating Company & http://www.firstenergycorp.com/ \\
\hline GPU Nuclear, Inc. & http://www2.gpu.com/home/about/operations/GPUNuclear.shtml \\
\hline Illumination Company (The) & http://www.illuminatingcompany.com/ \\
\hline Long Island Power Authority & http://www.lipa.state.ny.us/index.html \\
\hline Nebraska Public Power District & http://www.nppd.com/ \\
\hline New Century Energies & http://www.ncenergies.com \\
\hline New York Power Authority & http://www.nypa.gov/nypac.html \\
\hline Niagara Mohawk Power Corporation & http://www.nimo.com/ \\
\hline Northeast Utilities & http://www.nu.com/ \\
\hline Northern States Power Company & http://www.nspco.com/ \\
\hline Omaha Public Power District & http://www.oppd.com/ \\
\hline Pacific Gas and Electric Company & http://www.pge.com/ \\
\hline PECO Energy Company & http://www.peco.com/ \\
\hline Pennsylvania Power \& Light Company & http://www.ppl-inc.com/index.html \\
\hline Portland General Electric Company & http://www.pge-online.com/ \\
\hline Public Service Electric \& Gas Company & http://www.pseg.com/ \\
\hline Rochester Gas \& Electric Corporation & http://www.rge.com/ \\
\hline
\end{tabular}


Table 9. Nuclear Power Plant Operator Web Sites

Utility

Web Site

Sacramento Municipal Utility District

South Carolina Electric \& Gas Company

Southern California Edison Company

Southern Nuclear Operating Company (The)

STP Nuclear Operating Company

Tennessee Valley Authority

Toledo Edison Company

TXU Electric and Gas

Wisconsin Electric Power Company

Wisconsin Public Service Corporation

Wolf Creek Nuclear Operating Corporation

Vermont Yankee Nuclear Power Corporation

Yankee Atomic Electric Company http://www.smud.org/index.html

http://www.scana.com/sce\&g/default.htm

http://www.sce.com/

http://www.southernco.com/site/southernnuclear/

http://www.stpnoc.com/

http://www.tva.gov/

http://www.toledoedison.com/

http://www.txu.com/default.asp

http://www.wisconsinelectric.com/

http://www.wpsc.wpsr.com/

http://www.wcnoc.com/

http://www.vermontyankee.com/

http://www.yankee.com/ 


\section{REFERENCES}

1. Nuclear Plant O\&M Cost Data 1981-1998, Utility Data Institute, July 1999.

2. Nuclear Reactors Built, Being Built, or Planned: 1996, DOE/OSTI-8200-R60, U.S. Department of Energy, Office of Nuclear Energy, Science and Technology, August 1997.

3. Electrical World Directory of Electric Power Producers and Distributors, 107th Edition, McGraw-Hill, 1999.

4. "World List of Nuclear Power Plants", Nuclear News, p. 33, March 1999. 

NUREG/CR-6500, Rev. 1 ORNL/TM-13297/R1

Dist. Categories AN, CS, XG, 1S, 9L

\section{INTERNAL DISTRIBUTION}

$\begin{array}{cl}\text { 1. } & \text { A. R. Bush } \\ \text { 2. } & \text { W. C. Craddick } \\ \text { 3. } & \text { E. C. Fox } \\ \text { 4. } & \text { R. G. Gilliland } \\ \text { 5. } & \text { S. R. Greene } \\ \text { 6. } & \text { S. A. Hodge } \\ \text { 7. } & \text { G. T. Mays } \\ \text { 8. } & \text { S. R. McNeany } \\ \text { 9. } & \text { G. E. Michaels } \\ \text { 10. } & \text { D. L. Moses } \\ \text { 11. } & \text { W. P. Poore } \\ \text { 12. } & \text { C. E. Pugh }\end{array}$

1. A. R. Bush

2. W. C. Craddick

3. E. C. Fox

4. R. G. Gilliland

5. S. R. Greene

6. S. A. Hodge

7. G. T. Mays

E. Pugh
13-22.
R. L. Reid
23.
J. J. Simpson
24. R. E. Schilling
25-29.
V. S. White
30.
D. L. Williams, Jr.
31.
K. A. Williams
32.
Central Research Library
33. ORNL Y-12 Research Library
Document Reference Section
34-35. Laboratory Records Department
36. Laboratory Records, ORNL
37. ORNL Patent Office

\section{EXTERNAL DISTRIBUTION}

38. R. C. Brady, Office of Nuclear Reactor Regulation, U.S. Nuclear Regulation Commission, MS 012 E5, Washington, D.C. 20555-0001

39. R. T. Eynon, Energy Information Administration, U.S. Department of Energy, 1000 Independence Avenue, Washington, D.C. 20585

40. M. Fertel, Nuclear Energy Institute, 1776 I Street MW, Suite 400, Washington, D.C. 20006-3708

41. J. C. Geidl, Energy Information Administration, U.S. Department of Energy, 1000 Independence Avenue, Washington, D.C. 20585

42. R. L. Jones, VP Nuclear Power, Electric Power Research Institute, P.O. Box 10412, Palo Alto, CA 94303-0813

43. W. D. Magwood, IV, Office of Nuclear Energy, Science and Technology, U.S. Department of Energy, Washington, D.C. 20585

44. J. M. Stamos, Office of Nuclear Energy, Science and Technology, U.S. Department of Energy, Washington, D.C. 20585

45. R. S. Wood, Office of Nuclear Reactor Regulation, U.S. Nuclear Regulatory Commission, MS 011 F1, Washington, D.C. 20555-0001

46. Office of the ORNL Site Manager, Department of Energy, Oak Ridge National Laboratory, P.O. Box 2008, Oak Ridge, TN 37831

47-48. Office of Scientific and Technical Information, P.O. Box 62, Oak Ridge, TN 37831 



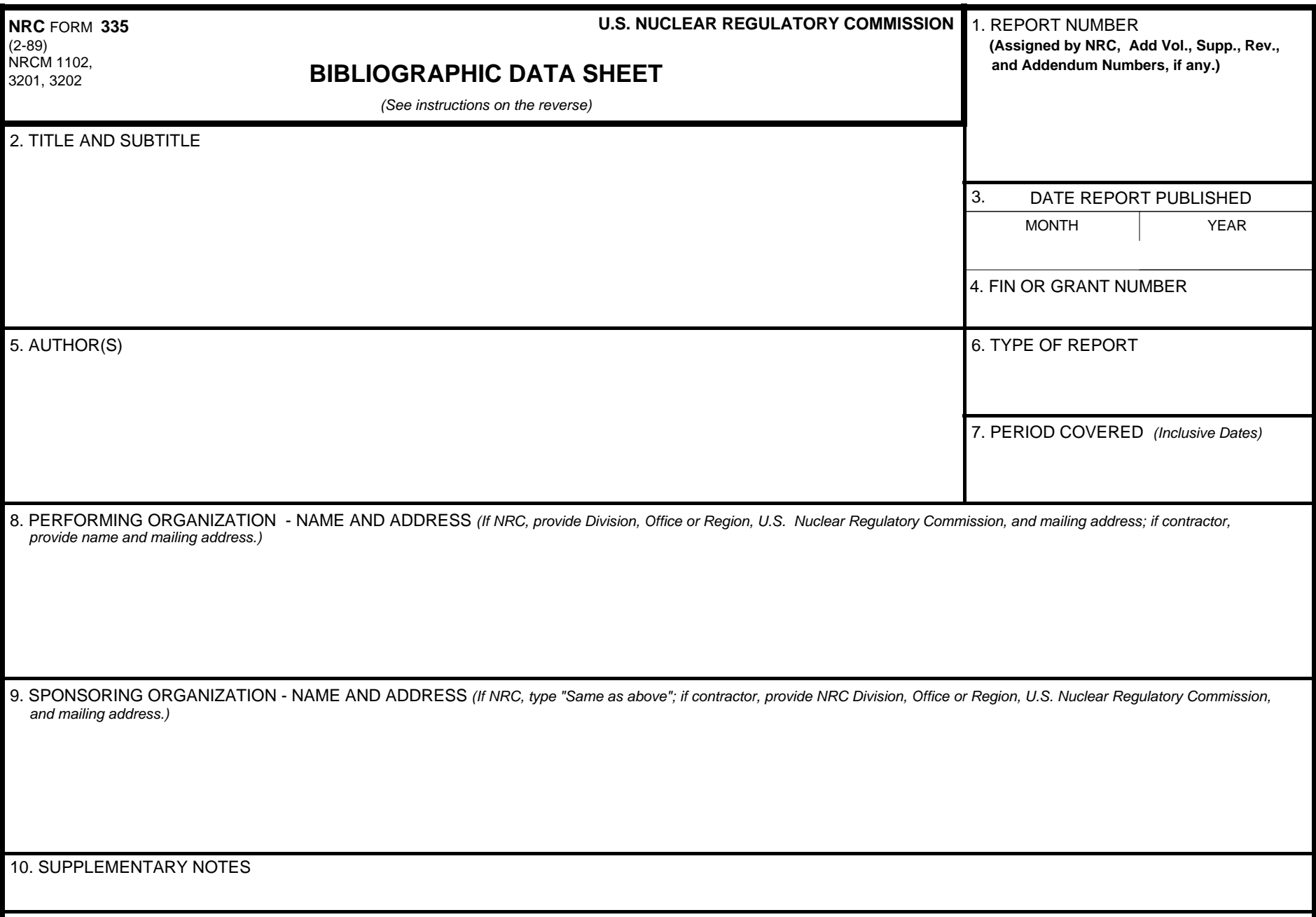

11. ABSTRACT (200 words or less)

Commercial nuclear power plants in this country can be owned by a number of separate entities, each with varying ownership portions. Each of these owners may, in turn, have a parent/subsidiary relationahip to other companies. In addition, the operator of the plant may be a different entity as well. This report provides a compilation on the owners/operators for all commercial power reactors in the United States. While the utility industry is currently experiencing changes in organizational structure which may affect nuclear plant ownership, the data in this report is current as of November 1999. The report is divided into the following sections representing different aspects of nuclear plant ownership.

- Nuclear Power Plant Percentage Ownership Ordered by Plant Name

- Nuclear Power Plant Percentage Ownership Ordered by Utility Name

- Utility/Company Relationships Ordered by Parent/Holding Company

- Utility/Company Relationships Ordered by Subsidiary

- Nuclear Power Plants Listed by Operator

- Nuclear Power Plants Listed by Plant Name

- Nuclear Power Plants Listed by State

12. KEY WORDS/DESCRIPTORS (List words or phrases that will assist researchers in locating the report.)

Nuclear Power Plants

Owners (Ownership)

Utilities

Operators

Holding Companies

\begin{tabular}{|c|}
$\begin{array}{c}\text { 13. AVAILABILITY STATEMENT } \\
\text { Unlimited }\end{array}$ \\
\hline $\begin{array}{c}\text { 14. SECURITY CLASSIFICATION } \\
\text { (This Page) } \\
\text { Unclassified }\end{array}$ \\
$\begin{array}{c}\text { (This Report) } \\
\text { Unclassified }\end{array}$ \\
\hline $\begin{array}{l}\text { 15. NUMBER OF PAGES } \\
\text { 16. PRICE }\end{array}$ \\
\hline
\end{tabular}


